\title{
Image inverse d'un $\mathcal{D}$-module et polygone de Newton
}

\author{
(Inverse Image of a $\mathcal{D}$-module and Newton Polygon) \\ YVES LAURENT ${ }^{1}$ et ZOGHMAN MEBKHOUT ${ }^{2}$ \\ ${ }^{1}$ Université de Grenoble, Institut Fourier, UMR 5584 CNRS/UJF, BP 74, 38042 St Martin \\ d'Hères Cedex, France.e-mail: yves.laurent@ujf-grenoble.fr \\ ${ }^{2}$ Université de Paris 7 UFR de Mathématiques, 175 rue de Chevaleret, 75013 Paris, France. \\ e-mail:mebkout@math.jussieu.fr
}

(Received: 19 September 2000)

\begin{abstract}
We show that, under conditions about the microcharacteristic variety of a coherent $\mathcal{D}$-module, the Cauchy problem is well-posed in the spaces of formal power series with Gevrey growth. We deduce that the filtration of the Irregularity Sheaf of a holonomic $\mathcal{D}$-module, which we defined in a previous work, is preserved under inverse image if some rather general geometric conditions are fullfilled.
\end{abstract}

Mathematics Subject Classification (2000). 35A27.

Key words: $\mathcal{D}$-modules, irregularity, index, holonomic, Gevrey.

\section{Introduction}

Soient $\left(X, \mathcal{O}_{X}\right)$ une variété analytique complexe, $\mathcal{D}_{X}$ le faisceau des opérateurs différentiels d'ordre localement fini et $Z$ une sous-variété non singulière de $X$. Si $Z$ est non caractéristique pour un $\mathcal{D}_{X}$-module cohérent $\mathcal{M}$ le $\mathcal{D}_{Z}$-module induit $\mathcal{M}_{Z}$ est cohérent et le morphisme de Cauchy-Kowalevska

$$
\left.\mathbb{R} \mathcal{H} \operatorname{om}_{\mathcal{D}_{X}}\left(\mathcal{M}, \mathcal{O}_{X}\right)\right|_{Z} \rightarrow \mathbb{R} \mathcal{H} \operatorname{om}_{\mathcal{D}_{Z}}\left(\mathcal{M}_{Z}, \mathcal{O}_{Z}\right)
$$

est un isomorphisme [3]. Lorsque $\mathcal{M}$ est holonome il existe une partie localement finie $F$ de $X$, telle que par tout point de $X-F$ il passe au moins une hypersurface non caractéristique.

Si $Y$ est une hypersurface de $X$ le complété formel $\mathcal{O}_{X \hat{Y} Y}$ est une $\mathcal{D}_{X}$-module à gauche et l'on a la formule de dualité [10, 2.2.1.3]:

$$
\mathbb{R} \mathcal{H} \operatorname{om}_{\mathcal{D}_{X}}\left(\mathbb{R} \Gamma_{[Y]}(\mathcal{M}), \mathcal{O}_{X}\right) \simeq \mathbb{R} \mathcal{H} \operatorname{om}_{\mathcal{D}_{X}}\left(\mathcal{M}, \mathcal{O}_{X \hat{\mid}}\right)
$$

où $\mathbb{R} \Gamma_{[Y]}(\mathcal{M})$ est le complexe de cohomologie locale algébrique. Le problème de Cauchy pour un module à valeur dans les fonctions formelles se ramène au problème de Cauchy pour son complexe de cohomologie locale algébrique à valeur dans les fonctions holomorphes. D'autre part le complexe de cohomologie locale commute à l'image inverse. Si bien que si $\mathcal{M}$ est un $\mathcal{D}_{X}$-module cohérent tel que 
son complexe de cohomologie locale est à cohomologie cohérente et que $Z$ est une sous-variété non caractéristique pour les deux faisceaux de cohomologie locale le morphisme de Cauchy-Kowalevska

$$
\left.\mathbb{R} \mathcal{H} \operatorname{om}_{\mathcal{D}_{X}}\left(\mathcal{M}, \mathcal{O}_{X \mid \hat{Y}}\right)\right|_{Z} \rightarrow \mathbb{R} \mathcal{H} \operatorname{om}_{\mathcal{D}_{Z}}\left(\mathcal{M}_{Z}, \mathcal{O}_{Z \mid \hat{Z} \cap \cap Y}\right)
$$

est un isomorphisme. Lorsque $\mathcal{M}$ est holonome, les faisceaux de cohomologie locale sont encore holonomes et il existe donc encore une partie localement finie $F^{\prime}$ de $X$ en dehors de laquelle passe par tout point au moins une hypersurface non caractéristique pour $\mathcal{M}$ et $\mathbb{R} \Gamma_{[Y]}(\mathcal{M})$.

Par définition [10], l'irrégularité d'un module holonome $\mathcal{M}$ est le complexe des solutions de $\mathcal{M}$ dans le quotient de $\mathcal{O}_{X \hat{Y} Y}$ par $\mathcal{O}_{X}$, et ce qui précède montre que l'irrégularité commute à l'image inverse par les sous-variétés non caractéristiques pour $\mathcal{M}$ et $\mathbb{R} \Gamma_{[Y]}(\mathcal{M})$.

Les problèmes de Cauchy à valeurs dans les fonctions holomorphes et à valeurs dans les fonctions formelles le long d'une hypersurface sont deux cas extrêmes des problèmes de Cauchy à valeurs dans les fonctions de classe de Gevrey le long de $Y$ d'ordre $r \in[1, \infty]$, fonctions avec lesquelles nous avons pu définir une filtration de l'irrégularité [9].

Dans cet article nous nous proposons de montrer que le résultat précédent reste vrai pout tout $r$ : Si $\mathcal{M}$ est un $\mathcal{D}_{X}$ module holonome il passe en dehors d'une partie localement finie de $X$ une hypersurface $Z$ telle que le problème de Cauchy à valeurs dans les fonctions de classe de Gevrey le long de $Y$ d'ordre $r \in[1, \infty]$ est bien posé, i.e. le morphisme

$$
\left.\mathbb{R} \mathcal{H} \operatorname{om}_{\mathcal{D}_{X}}\left(\mathcal{M}, \mathcal{O}_{X \mid Y}\{r\}\right)\right|_{Z} \rightarrow \mathbb{R} \mathcal{H} \operatorname{om}_{\mathcal{D}_{Z}}\left(\mathcal{M}_{Z}, \mathcal{O}_{Z \mid Z \cap Y}\{r\}\right)
$$

est un isomorphisme pour tout $r \in[1, \infty]$.

Ce problème de Cauchy avait déjà été étudié dans [5], plus exactement on considérait non pas les fonctions de classe de Gevrey mais le problème dual avec les faisceaux de cohomologie locale de classe de Gevrey $\mathcal{B}_{Y \mid X}\{r\}$. On montrait que le problème de Cauchy est bien posé dans le faisceau $\mathcal{B}_{Y \mid X}\{r\}$ à condition de remplacer la variété caractéristique par une variété ' $\{r\}$-microcaractéristique' et de faire un changement de base $\mathcal{D}_{X} \rightarrow \mathcal{D}_{X \mid Y}\{r\}$ pour définir l'image inverse. On obtenait ainsi un isomorphisme

$$
\left.\mathbb{R} \mathcal{H} \operatorname{om}_{\mathcal{D}_{X}}\left(\mathcal{M}, \mathcal{B}_{Y \mid X}\{r\}\right)\right|_{Z} \stackrel{\sim}{\longrightarrow} \mathbb{R} \mathcal{H} \operatorname{om}_{\mathcal{D}_{Z \mid Z \cap Y}\{r\}}\left(\mathcal{M}_{Z}\{r\}, \mathcal{B}_{Z \mid Z \cap Y}\{r\}\right)
$$

Le faisceau $\mathcal{D}_{X \mid Y}\{r\}$ est un faisceau d'opérateurs différentiels d'ordre infini à coefficients dans $\mathcal{O}_{X \mid Y}\{r\}$. Le problème est qu'en général l'image inverse ne commute pas à ce changement de base.

En fait, nous étudions le problème plus général de l'image inverse par une application $\varphi: Z \rightarrow X$ transverse à $Y$ et nous montrons que si $\mathcal{M}$ est holonome et si l'application $\varphi$ est non $\{r\}$-microcaractéristique pour tout $r$, alors on a bien com- 
mutation entre image inverse et changement de base $\mathcal{D}_{X} \rightarrow \mathcal{D}_{X \mid Y}\{r\}$ pour tout $r$ (corollaire 2.2.3.).

Dans le cas holonome, la condition ' $\varphi$ est non $\{r\}$-microcaractéristique' ne fait intervenir qu'un nombre fini de variétés lagrangiennes lorsque $r$ varie et donc elle est suffisamment générique pour qu'il existe des hypersurfaces non $\{r\}$-microcaractéristique' pour tout $r$ en tout point sauf un ensemble localement fini.

Nous utilisons d'une part le problème de Cauchy pour les $\mathcal{D}_{X \mid Y}\{r\}$ à valeurs dans les faisceaux de cohomologie locale de classe de Gevrey $\mathcal{B}_{Y \mid X}\{r\}$ [5] et d'autre part la finitude des pentes [6] en supposant que $Y$ est non singulière.

Ce résultat a pour conséquence que la filtration Gevrey du faisceau d'irrégularité d'un $\mathcal{D}_{X}$-module holonome le long d'une hypersurface [10] commute à la restriction assez générale.

Un autre résultat important que nous obtenons est que sous les conditions précédentes, les pentes du module $\mathcal{M}$ et celles de son image inverse par $\varphi$ sont les mêmes (2.2.9), en fait c'est même le polygone de Newton tel qu'il a été défini dans [9] qui est conservé.

\section{Problème de Cauchy dans les espaces de type Gevrey}

\subsection{VARIÉTÉS MICROCARACTÉRISTIQUES (RAPPELS)}

(Les variétés microcaractéristiques et les 'pentes' d'un $\mathcal{D}_{X}$-module ont été définies dans [5] et [6]. Ces définitions ont été reprises dans [9] et nous donnons ici seulement un résumé très rapide.)

Dans tout le chapitre, on considère une variété analytique complexe $X$ de dimension $n$ et une sous-variété lisse $Y$ de $X$. On note $\Lambda=T_{Y}^{*} X$ le fibré conormal à $Y$ dans $X$ et $T^{*} \Lambda$ le fibré cotangent à $\Lambda, \mathcal{O}_{T^{*} \Lambda}$ le faisceau des fonctions holomorphes sur $T^{*} \Lambda$ et $\mathcal{O}_{\left[T^{*} \Lambda\right]}$ le sous-faisceau de $\mathcal{O}_{T^{*} \Lambda}$ des fonctions qui sont polynomiales dans les fibres de $\pi: T^{*} \Lambda \rightarrow Y$.

Le faisceau $\mathcal{D}_{X \mid Y}$ des opérateurs différentiels à coefficients holomorphes définis au voisinage de $Y$ est muni de deux filtrations canoniques. La première est la filtration usuelle par l'ordre des opérateurs que nous noterons $\left(\mathcal{D}_{X, m}\right)_{m \geqslant 0}$ et la seconde est définie [4] par:

$$
V_{k} \mathcal{D}_{X}=\left\{\left.P \in \mathcal{D}_{X}\right|_{Y} / \forall j \in \mathbb{Z}, \quad P \mathcal{I}_{Y}^{j} \subset \mathcal{I}_{Y}^{j-k}\right\}
$$

où $\mathcal{I}_{Y}$ est l'idéal de définition de $Y$ et $\mathcal{I}_{Y}^{j}=\mathcal{O}_{X}$ si $j \leqslant 0$.

Si $r$ est un nombre rationnel strictement supérieur à 1 d'écriture irréductible $r=p / q$ avec $p>q>1$, on définit les filtrations $F_{r}$ :

$$
F_{r}^{k} \mathcal{D}_{X}=\sum_{(p-q) m+q n=k} \mathcal{D}_{X, n} \cap V_{m} \mathcal{D}_{X}
$$


Pour tout $r>1$, le gradué associé $\operatorname{gr}_{F_{r}} \mathcal{D}_{X}$ est isomorphe au faisceau d'anneaux $\pi_{*} \mathcal{O}_{\left[T^{*} \Lambda\right]}$ [6]. Pour $r=1$, on pose encore $F_{1}^{k} \mathcal{D}_{X}=\mathcal{D}_{X, k}$ et pour $r=\infty, F_{\infty}^{k} \mathcal{D}_{X}=$ $V_{k} \mathcal{D}_{X}$ (mais dans ces deux cas le gradué n'est plus le même).

Soit $\left(x_{1}, \ldots, x_{n-p}, t_{1}, \ldots, t_{p}\right)$ un système de coordonnées locales de $X$ tel que

$$
Y=\{(x, t) \in X \mid t=0\} \quad \text { et } \quad \Lambda=\left\{(x, t, \xi, \tau) \in T^{*} X \mid t=0, \xi=0\right\} .
$$

On note $\left(x, \tau, x^{*}, \tau^{*}\right)$ les coordonnées correspondantes de $T^{*} \Lambda$. Dans un tel système de coordonnées un opérateur $\left.P \in \mathcal{D}_{X}\right|_{Y}$ s'écrit:

$$
P\left(x, t, D_{x}, D_{t}\right)=\sum p_{\alpha k l}(x) t^{l} D_{t}^{k} D_{x}^{\alpha}
$$

et il est dans $F_{r}^{N} \mathcal{D}_{X}$ si et seulement si $p(k-l)+q(|\alpha|+l) \leqslant N$ quand $p_{\alpha k l} \not \equiv 0$.

Alors la fonction

$$
\sigma_{r}(P)=\sum_{p(k-l)+q(\mid \alpha+l)=N} p_{\alpha k l}(x)\left(\tau^{*}\right)^{l}(-\tau)^{k}\left(x^{*}\right)^{\alpha}
$$

est bien définie sur $T^{*} \Lambda$ (i.e. est indépendante des coordonnées locales) et l'isomorphisme entre $\operatorname{gr}_{F_{r}} \mathcal{D}_{X}$ et $\pi_{*} \mathcal{O}_{\left[T^{*} \Lambda\right]}$ est donné par les fonctions $\sigma_{r}(P)$.

La fonction $\sigma_{r}(P)$ est bihomogène (i.e. homogène en $\left(x^{*}, \tau^{*}\right)$ et en $\left(x^{*}, \tau\right)$, sauf pour un nombre fini de valeurs de $r$, les pentes du polygone de Newton de $P$. Entre deux pentes, la fonction $\sigma_{r}(P)$ ne dépend pas de $r$.

Soit $\mathcal{M}$ un $\mathcal{D}_{X}$-module cohérent défini au voisinage de $Y$. Une bonne $F_{r}$-filtration de $\mathcal{M}$ est, par définition, une filtration sur $\mathcal{M}$ compatible avec la filtration $F_{r} \mathcal{D}_{X}$ et localement de type fini. On montre dans [6] que le gradué associé à une bonne $F_{r}$-filtration est un $g r_{F_{r}} \mathcal{D}_{X}=\pi_{*} \mathcal{O}_{\left[T^{*} \Lambda\right]}$ module cohérent qui définit un cycle analytique $\widetilde{\Sigma}_{\Lambda}(r)(\mathcal{M})$ sur $T^{*} \Lambda$.

Ce cycle est indépendant du choix de la bonne filtration, c'est le cycle microcaractéristique de type $(r)$ de $\mathcal{M}$, son support $\Sigma_{\Lambda}(r)(\mathcal{M})$ est la variété microcaractéristique.

Cette variété microcaractéristique est $r$-homogène c'est-à-dire invariante par l'action de $\mathbb{C}^{*}$ sur $T^{*} \Lambda$ qui s'écrit en coordonnées

$$
H_{r}(\lambda):\left(x, \tau, x^{*}, \tau^{*}\right) \rightarrow\left(x, \lambda^{p} \tau, \lambda^{q} x^{*}, \lambda^{q-p} \tau^{*}\right)
$$

Ces cycles sont en nombre fini pour un module donné:

THÉORÈME 1.1.1 [6, théorème 3.4.1]. Soit $\mathcal{M}$ un $\mathcal{D}_{X}$-module cohérent défini au voisinage de $Y$. Il existe (localement sur $Y$ ) une suite finie de rationnels $r_{0}=1<r_{1}<\cdots<r_{N}<r_{N+1}=+\infty$ telle que $\Sigma_{\Lambda}(r)(\mathcal{M})$ soit indépendant de $r$ dans chacun des intervalles ouverts $] r_{i}, r_{i+1}[$.

Si la variété $\Sigma_{\Lambda}(r)(\mathcal{M})$ est indépendante de $r$ sur un intervalle, elle est $r$-homogène pour tout $r$. Dans ce cas on dit qu'elle est bihomogène. Inversement on montre dans [7] que si $\Sigma_{\Lambda}\left(r_{0}\right)(\mathcal{M})$ est bihomogène alors $\Sigma_{\Lambda}(r)(\mathcal{M})$ est indépendant de $r$ au voisinage de $r_{0}$. 
Il y a donc deux familles finies distinctes de variétés microcaractéristiques, celles qui correspondent à l'un des nombres $r_{1}, \ldots, r_{N}$, elles sont $r_{i}$-homogène pour la pente $r_{i}$ correspondante, et celles qui correspondent à un intervalle entre deux pentes et sont bihomogènes.

DÉFINITION 1.1.2. Pour tout nombre rationnel $r>1$ on définit $I_{Y}(r)(\mathcal{M})$ comme l'adhérence dans $Y$ de la projection par $T^{*} \Lambda \rightarrow Y$ de la réunion des composantes irréductibles non bihomogènes de $\Sigma_{\Lambda}(r)(\mathcal{M})$.

On dit que $r$ est une pente de $\mathcal{M}$ en $y \in Y$ si $y \in I_{Y}(r)(\mathcal{M})$.

Pour simplifier les énoncés, on étend cette définition aux nombres réels en posant qu'un réel non rationnel n'est jamais une pente. D'autre part on considère toujours que 1 et $+\infty$ sont des pentes.

Puisque le nombre de pentes est fini, pour tout $r$ il existe $\varepsilon_{0}$ tel qu'il n'y ait pas de pentes dans les intervalles $] r-\varepsilon_{0}, r[$ et $] r, r+\varepsilon_{0}\left[\right.$. On pose $\widetilde{C} h_{\Lambda}\{r\}(\mathcal{M})$ $=\widetilde{\Sigma}_{\Lambda(r+\varepsilon)}(\mathcal{M})$ et $\widetilde{C h}_{\Lambda}(r)(\mathcal{M})=\widetilde{\Sigma}_{\Lambda(r-\varepsilon)}(\mathcal{M})$ pour $\varepsilon>0$ assez petit. On définit encore les variétés correspondant à ces cycles $C h_{\Lambda}(r)(\mathcal{M})$ et $C h_{\Lambda}\{r\}(\mathcal{M})$.

Si $r$ n'est pas une pente, les variétés $\Sigma_{\Lambda}(r)(\mathcal{M}), C h_{\Lambda}(r)(\mathcal{M})$ et $C h_{\Lambda}\{r\}(\mathcal{M})$ sont confondues. Si $r$ est une peute, on peut montrer [6] que $C h_{\Lambda}\{r\}(\mathcal{M})$ est le cône tangent à $\Sigma_{\Lambda}(r)(\mathcal{M})$ le long de la section nulle de $T^{*} \Lambda$ tandis que $C h_{\Lambda}(r)(\mathcal{M})$ s'obtient comme le cône tangent à l'infini (cf [9] pour un énoncé plus précis).

On montre [6, Propositions 3.5.2. et 3.5.3] que toutes les variétés microcaractéristiques $\Sigma_{\Lambda}(r)(\mathcal{M})$ et donc $C h_{\Lambda}(r)(\mathcal{M})$ et $C h_{\Lambda}\{r\}(\mathcal{M})$ sont involutives dans $T^{*} \Lambda$. De plus, si $\mathcal{M}$ est un module holonome elles sont lagrangiennes [6, Corollaire 4.1.2].

Les variétés $C h_{\Lambda}(r)(\mathcal{M})$ et $C h_{\Lambda}\{r\}(\mathcal{M})$ sont toujours bihomogènes et en particulier sont homogènes dans les fibres de $T^{*} \Lambda \rightarrow \Lambda$ et ont donc des propriétés géométriques analogues à celles des variétés caractéristiques. Par contre cela n'est pas vrai pour les variétés $\Sigma_{\Lambda}(r)(\mathcal{M})$ quand $r$ est une pente.

\subsection{OPÉRATEURS DIFFÉRENTIELS D’ORDRE INFINI}

Comme nous l'avons annoncé dans l'introduction, nous allons utiliser les images inverses de $\mathcal{D}$-modules qui dépendent de l'indice de croissance $r$ de [5] pour ensuite éliminer cette dépendance. Ces images inverses se définissent à partir de faisceaux d'opérateurs différentiels d'ordre infini particuliers dont nous allons rappeler la définition.

Le faisceau $\left.\mathcal{D}_{X}\right|_{Y}$ des opérateurs différentiels définis au voisinage de la variété $Y$ est muni de la $V$-filtration. Son complété pour cette filtration est noté dans [8] $\widehat{\mathcal{D}}_{X \mid Y}$, ici nous le noterons plutôt $\mathcal{D}_{X \mid Y}\{\infty\}$. Dans les coordonnées locales $(x, t)$ considérées plus haut, une section de $\mathcal{D}_{X \mid Y}\{\infty\}$ sur un ouvert $U$ de $Y$ s'écrit de manière unique comme une série formelle

$$
P\left(x, t, D_{x}, D_{t}\right)=\sum_{(\alpha, \beta, \gamma) \in \mathbb{N}^{n-p} \times \mathbb{N}^{p} \times \mathbb{N}^{p}} p_{\alpha, \beta, \gamma}(x) t^{\gamma} D_{x}^{\alpha} D_{t}^{\beta}
$$


où $p_{\alpha, \beta, \gamma}$ est holomorphe sur $U$ et où, pour chaque $k$, la somme pour $|\beta|-|\gamma|=k$ est finie.

Soit $r$ un nombre rationnel supérieur ou égal à 1 d'écriture irréductible $r=p / q$. Le faisceau $\mathcal{D}_{X \mid Y}\{r\}$ est le sous-faisceau de $\mathcal{D}_{X \mid Y}\{\infty\}$ dont les sections sur un ouvert $U$ sont les sections de $\mathcal{D}_{X \mid Y}\{\infty\}$ qui vérifient:

(1) $\exists N \in \mathbb{N}, p(|\beta|-|\gamma|)+q(|\alpha|+|\gamma|)>N \Rightarrow p_{\alpha, \beta, \gamma} \equiv 0$

(2) $\forall K \Subset U, \exists C_{0}>0, C_{1}>0$,

$$
\sup _{x \in K}\left|p_{\alpha, \beta, \gamma}(x)\right| \leqslant C_{0} C_{1}^{|\gamma|} \frac{(|\gamma| !)^{r-1}}{(|\beta| !)^{r}|\alpha| !}
$$

Le faisceau $\mathcal{D}_{X \mid Y}\{r\}$ est muni de la filtration $\left(F_{r}^{N} \mathcal{D}_{X \mid Y}\{r\}\right)_{N \in \mathbb{Z}}$ où $F_{r}^{N} \mathcal{D}_{X \mid Y}\{r\}$ est le sous-faisceau des sections qui vérifient (1) pour $N$ fixé.

Il est assez facile de montrer directement que ces définitions ne dépendent pas du choix des coordonnées locales, mais pour étudier les propriétés de ces faisceaux, il est préférable de les considérer comme un cas particulier des constructions de [5].

Dans [5] (voir aussi [6]), on a défini une famille $\mathcal{E}_{\Lambda}^{2}(r, s)$ de faisceaux sur $T^{*} \Lambda$ (les opérateurs '2-microdifférentiels') puis la famille $\mathcal{D}_{\Lambda}^{2}(r, s)$ de faisceaux sur $\Lambda$ par restriction à la section nulle de $\Lambda$ de $T^{*} \Lambda$, c'est-à-dire $\mathcal{D}_{\Lambda}^{2}(r, s)=\left.\mathcal{E}_{\Lambda}^{2}(r, s)\right|_{\Lambda}$. La restriction à la section nulle $Y$ de $\Lambda$ du faisceau $\mathcal{D}_{\Lambda}^{2}(r, s)$ ne dépend pas de $r$ et on a $\mathcal{D}_{X \mid Y}\{r\}=\left.\mathcal{D}_{\Lambda}^{2}(r, r)\right|_{Y}$.

On a en particulier $\mathcal{D}_{X \mid Y}\{1\}=\left.\mathcal{D}_{X}\right|_{Y}$ et, d'après [5], si $r \geqslant s \geqslant 1$ le faisceau d'anneaux $\mathcal{D}_{X \mid Y}\{r\}$ est plat sur $\mathcal{D}_{X \mid Y}\{s\}$.

La filtration $V$ est bien définie sur $\mathcal{D}_{X \mid Y}\{\infty\}$ donc sur $\mathcal{D}_{X \mid Y}\{r\}$ pour tout $r$. A partir de cette filtration et de la filtration $F_{r}$ on peut étendre à $\mathcal{D}_{X \mid Y}\{r\}$ la filtration $F_{S}$ si $s \geqslant r$ par une formule analogue à (1.1.1). On peut aussi remarquer que la famille $\mathcal{D}_{X \mid Y}\{r\}$ est croissante en $r$ et définir la filtration de la manière suivante:

$$
F_{s}^{N} \mathcal{D}_{X \mid Y}\{r\}=F_{s}^{N} \mathcal{D}_{X \mid Y}\{s\} \cap \mathcal{D}_{X \mid Y}\{r\} .
$$

Les définitions du paragraphe 1.1 s'étendent à $\mathcal{D}_{X \mid Y}\{r\}$ à condition de se restreindre à l'intervalle $] r, \infty$ ]. Plus précisément, le gradué associé à la filtration $F_{S}$ est isomorphe à $\pi_{*} \mathcal{O}_{\left[T^{*} \Lambda\right]}$ muni de la graduation correspondante, il ne dépend pas de $r$. Par contre, pour $s=r$ il s'identifie au faisceau des fonctions qui s'écrivent en coordonnées locales comme des sommes finies de séries:

$$
\sum_{p(|\beta|-|\gamma|)+q(|\alpha|+|\gamma|)=N} p_{\alpha, \beta, \gamma}(x)\left(-\tau^{*}\right)^{\gamma}\left(x^{*}\right)^{\alpha} \tau^{\beta}
$$

$\operatorname{avec} \sup _{K}\left|p_{\alpha, \beta, \gamma}\right|<C_{0} C_{1}^{|\gamma|}$.

Ces séries sont convergentes dans un domaine

$$
\left|\tau^{*}\right|\left|x^{*}\right|^{r-1}<C, \quad\left|\tau^{*}\right|^{r}|\tau|^{r-1}<C
$$

c'est-à-dire dans un voisinage de $Y$ conique pour $H_{r}$ et le gradué s'identifie donc à un sous-faisceau de $\left.\mathcal{O}_{T^{*} \Lambda}\right|_{Y}$. 
Ce fait est à rapprocher du cas $r=1$ où $F_{1}$ est la filtration usuelle par l'ordre des opérateurs et où le gradué associé est le faisceau des fonctions holomorphes sur $T^{*} X$ polynomiales dans les fibres et définies au voisinage de $Y$.

D'après [5], ces filtrations sont 'noethériennes' comme dans le cas de $\mathcal{D}_{X}$ et donc si $\mathcal{M}$ est un $\mathcal{D}_{X \mid Y}\{r\}$-module cohérent muni d'une bonne $F_{S}$-filtration, le gradué associé est un $\mathcal{O}_{\left[T^{*} \Lambda\right]}$-module cohérent si $s>r$ et un $\mathcal{O}_{T^{*} \Lambda}$-module cohérent défini sur un domaine du type 1.2.2 si $r=s$. Le cycle analytique associé à ce gradué est indépendant du choix de la bonne filtration. Pour $s>r$ on le note encore $\widetilde{\Sigma}_{\Lambda}(s)(\mathcal{M})$ tandis que pour $s=r$ on a seulement un germe de variété sur $Y$ que l'on note $\widetilde{\Sigma}_{\Lambda}^{0}(s)(\mathcal{M})$.

LEMME 1.2.1. Si $s>r^{\prime} \geqslant r$ et si $\mathcal{M}$ est un $\mathcal{D}_{X \mid Y}\{r\}$-module cohérent, on a:

$$
\begin{aligned}
& \widetilde{\Sigma}_{\Lambda}(s)\left(\mathcal{D}_{X \mid Y}\left\{r^{\prime}\right\} \otimes_{\mathcal{D}_{X \mid Y}\{r\}} \mathcal{M}\right)=\widetilde{\Sigma}_{\Lambda}(s)(\mathcal{M}) \\
& \widetilde{\Sigma}_{\Lambda}^{0}(s)\left(\mathcal{D}_{X \mid Y}\{s\} \otimes_{\mathcal{D}_{X \mid Y}\{r\}} \mathcal{M}\right)=\left.\left(\widetilde{\Sigma}_{\Lambda}(s)(\mathcal{M})\right)\right|_{Y}
\end{aligned}
$$

où $\left.(\Sigma)\right|_{Y}$ désigne le germe de la variété $\Sigma$ sur $Y$.

Démonstration. Par récurrence sur le nombre de générateurs de $\mathcal{M}$, on se ramène au cas d'un module à un seul générateur, c'est-à-dire au quotient de $\mathcal{D}_{X \mid Y}\{r\}$ par un idéal cohérent $\mathcal{I}$. Il suffit dans ce cas de montrer que:

$$
g r_{F_{s}}\left(\mathcal{D}_{X \mid Y}\left\{r^{\prime}\right\} \mathcal{I}\right)=g r_{F_{s}}\left(\mathcal{D}_{X \mid Y}\left\{r^{\prime}\right\}\right) g r_{F_{s}}(\mathcal{I})
$$

Supposons tout d'abord $s>r^{\prime}$, on a $\operatorname{gr}_{F_{s}}\left(\mathcal{D}_{X \mid Y}\left\{r^{\prime}\right\}\right)=g r_{F_{s}}\left(\mathcal{D}_{X \mid Y}\{r\}\right)$ et on applique le lemme 2.6.0. de [5].

Si $s=r^{\prime}$, on considère le faisceau $\mathcal{D}_{X \mid Y}(s)$ qui en coordonnées est le sous-faisceau de $\mathcal{D}_{X \mid Y}\{s\}$ des opérateurs tels que si $s=p / q$ et

$$
P\left(x, t, D_{x}, D_{t}\right)=\sum_{(\alpha, \beta, \gamma) \in \mathbb{N}^{n-p} \times \mathbb{N}^{p} \times \mathbb{N}^{p}} p_{\alpha, \beta, \gamma}(x) t^{\gamma} D_{x}^{\alpha} D_{t}^{\beta}
$$

alors pour $p(|\beta|-|\gamma|)+q(|\alpha|+|\gamma|)$ fixé, il y ait un nombre fini de $p_{\alpha, \beta, \gamma}$ non nuls.

On a $\operatorname{gr}_{F_{s}}\left(\mathcal{D}_{X \mid Y}(s)\right)=\operatorname{gr}_{F_{s}}\left(\mathcal{D}_{X \mid Y}\{r\}\right)$ donc en appliquant à nouveau le lemme 2.6.0. de [5] on obtient

$$
\operatorname{gr}_{F_{s}}\left(\mathcal{D}_{X \mid Y}(s) \mathcal{I}\right)=\operatorname{gr}_{F_{s}}\left(\mathcal{D}_{X \mid Y}(s)\right) \operatorname{gr}_{F_{s}}(\mathcal{I})
$$

D'autre part la filtration $F_{s}$ est zariskienne au sens de [13] sur $\mathcal{D}_{X \mid Y}(s)$ et $\mathcal{D}_{X \mid Y}\{s\}$ d'après le théorème 2.5.1. de [5] donc

$$
g r_{F_{s}}\left(\mathcal{D}_{X \mid Y}\{s\} \mathcal{J}\right)=g r_{F_{s}}\left(\mathcal{D}_{X \mid Y}\{s\}\right) g r_{F_{s}}(\mathcal{J})
$$

pour tout idéal cohérent $\mathcal{J}$ de $\mathcal{D}_{X \mid Y}(s)([1])$.

Lorsque $s>r$, on peut définir les cycles $\widetilde{C h}(s)(\mathcal{M})$ et $\widetilde{C} h_{\Lambda}\{s\}(\mathcal{M})$ pour un $\mathcal{D}_{X \mid Y}\{r\}$-module comme on l'a fait pour les $\mathcal{D}_{X}$-modules en posant 
$\widetilde{C} h_{\Lambda}\{s\}(\mathcal{M})=\widetilde{\Sigma}_{\Lambda(s+\varepsilon)}(\mathcal{M})$ et $\widetilde{C} h_{\Lambda}(s)(\mathcal{M})=\widetilde{\Sigma}_{\Lambda(s-\varepsilon)}(\mathcal{M})$ pour $\varepsilon>0$ assez petit. Lorsque $r=s$, la même formule définit $\widetilde{C} h_{\Lambda}\{s\}(\mathcal{M})$ mais évidemment $\widetilde{C h}(s)(\mathcal{M})$ n'est pas défini.

Le théorème 1.1.1 est encore vrai lorsqu'on se limite à l'intervalle $] r, \infty]$ et les variétés $\Sigma_{\Lambda}(s)(\mathcal{M}), C h_{\Lambda}(s)(\mathcal{M})$ et $C h_{\Lambda}\{s\}(\mathcal{M})$ supports des cycles correspondants sont involutives et même lagrangiennes quand $\mathcal{M}$ est holonome. Ces résultats se montrent exactement comme les résultats analogues pour les $\mathcal{D}_{X}$-modules dans [6].

Le cycle $\widetilde{C} h_{\Lambda}\{s\}(\mathcal{M})$ est le cône tangent le long de $\Lambda$ à $\widetilde{\Sigma}_{\Lambda}(s)(\mathcal{M})$ où à $\widetilde{\Sigma}_{\Lambda}^{0}(s)(\mathcal{M})$. (Remarquons que ce cône tangent ne dépend que des composantes irréductibles de $\widetilde{\Sigma}_{\Lambda}(s)(\mathcal{M})$ qui rencontrent $Y$ et qu'il peut donc se calculer à partir de $\widetilde{\Sigma}_{\Lambda}^{0}(s)(\mathcal{M})$ ).

\subsection{IMAGE INVERSE D'UN MODULE}

On considère une sous-variété $Z$ de $X$ transverse à $Y$. Alors $T_{Y \cap Z}^{*} Z \simeq\left(T_{Y}^{*} X\right)$ $\times_{Y}(Z \cap Y)$ est une sous-variété lisse de $\Lambda=T_{Y}^{*} X$ que nous noterons $\Lambda^{\prime}$. Le plongement $\Lambda^{\prime} \hookrightarrow \Lambda$ définit les morphismes:

$$
T^{*} \Lambda^{\prime} \stackrel{\varrho}{\longleftarrow}\left(T^{*} \Lambda\right) \times \Lambda \Lambda^{\prime} \stackrel{\varpi}{\longrightarrow} T^{*} \Lambda .
$$

On peut choisir des coordonnées locales $\left(x^{\prime}, x^{\prime \prime}, t\right)$ de $X$ telles que $Y$ soit donnée par l'équation $\{t=0\}$ et $Z$ par $\left\{x^{\prime}=0\right\}$. On a alors

$$
\begin{aligned}
& \Lambda^{\prime}=\left\{\left(x^{\prime}, x^{\prime \prime}, \tau\right) \in \Lambda \mid x^{\prime}=0\right\}, \\
& T_{\Lambda^{\prime}}^{*} \Lambda=\left\{\left(x^{\prime}, x^{\prime \prime}, \tau, x^{\prime *}, x^{\prime *}, \tau^{*}\right) \in T^{*} \Lambda \mid x^{\prime}=0, x^{\prime \prime *}=0, \tau^{*}=0\right\}
\end{aligned}
$$

et $\varrho$ et $\varpi$ sont données par:

$$
\begin{aligned}
& \varrho\left(x^{\prime \prime}, \tau, x^{\prime *}, x^{\prime \prime *}, \tau^{*}\right)=\left(x^{\prime \prime}, \tau, x^{\prime \prime *}, \tau^{*}\right), \\
& \varpi\left(x^{\prime \prime}, \tau, x^{\prime *}, x^{\prime \prime *}, \tau^{*}\right)=\left(0, x^{\prime \prime}, \tau, x^{\prime *}, x^{\prime \prime *}, \tau^{*}\right) .
\end{aligned}
$$

Plus généralement, on peut considérer une variété analytique complexe $Z$ et une application analytique $\varphi: Z \rightarrow X$. Le fibré conormal à $\varphi$, que l'on note $T_{Z}^{*} X$, est le noyau de $\left(T^{*} X\right) \times_{X} Z \rightarrow T^{*} Z$. Nous supposerons ici que $\varphi$ est transverse à $Y$, c'est-à-dire que $T_{Z}^{*} X \cap\left(T_{Y}^{*} X\right) \times_{X} Z=\{0\}$. On pose alors $Y^{\prime}=\varphi^{-1}(Y)$ et il y a un isomorphisme $T_{Y^{\prime}}^{*} X \simeq\left(T_{Y}^{*} X\right) \times_{Y} Y^{\prime}$. On note $\Lambda^{\prime}$ ce fibré et l'application $\Lambda^{\prime} \rightarrow \Lambda$ définit les morphismes $\varrho$ et $\varpi$ comme précédemment.

DÉFINITION 1.3.1. Le morphisme $\varphi$ est non $\{r\}$-microcaractéristique (resp. $(r)$-) pour $\mathcal{M}$ le long de $Y$ si l'intersection de $C h_{\Lambda}\{r\}(\mathcal{M})\left(\operatorname{resp} . C h_{\Lambda}(r)(\mathcal{M})\right)$ et de $T_{\Lambda^{\prime}}^{*} \Lambda$ est contenue dans la section nulle de $T^{*} \Lambda$.

Les variétés considérées étant homogènes dans les fibres de $T^{*} \Lambda$, cette définition est, comme dans le cas des variétés caractéristiques, équivalente à la finitude du 
morphisme $\varrho$ sur $\varpi^{-1} C h_{\Lambda}\{r\}(\mathcal{M})$ ou $\varpi^{-1} C h_{\Lambda}(r)(\mathcal{M})$. Lorsque $\varphi$ est le plongement d'une sous-variété $Z$ de $X$, on dit encore que $Z$ est non microcaractéristique.

L'image inverse d'un $\mathcal{D}_{X}$-module cohérent $\mathcal{M}$ par $\varphi$ est, par définition, l'image inverse au sens des $\mathcal{O}_{X}$-modules c'est-à-dire:

$$
\varphi^{*} \mathcal{M}=\mathcal{O}_{Z} \otimes_{\varphi^{-1} \mathcal{O}_{X}} \varphi^{-1} \mathcal{M} .
$$

Elle est munie canoniquement d'une structure de $\mathcal{D}_{Z}$-module et si $\varphi$ n'est pas caractéristique, $\varphi^{*} \mathcal{M}$ est un $\mathcal{D}_{Z}$-module cohérent. De manière analogue, on pose:

\section{DÉFINITION 1.3.2.}

$$
\varphi^{*} \mathcal{M}\{r\}=\mathcal{O}_{Z} \otimes_{\varphi^{-1} \mathcal{O}_{X}} \varphi^{-1}\left(\mathcal{D}_{X \mid Y}\{r\} \otimes_{\mathcal{D}_{X}} \mathcal{M}\right)
$$

Par définition, on a $\varphi^{*} \mathcal{M}\{1\}=\varphi^{*} \mathcal{M}$. Le faisceau $\varphi^{*} \mathcal{M}\{r\}$ est muni canoniquement d'une structure de $\mathcal{D}_{Z \mid Y^{\prime}}\{r\}$-module et on a [5, théorème 2.10.4]:

PROPOSITION 1.3.3. Si $Z$ est non $\{r\}$-microcaractéristique pour $\mathcal{M}$, le faisceau $\varphi^{*} \mathcal{M}\{r\}$ est un $\mathcal{D}_{Z \mid Y^{\prime}}\{r\}$-module cohérent.

Ce résultat se démontre à l'aide de théorèmes du type 'préparation de Weierstrass' et 'division' comme dans le cas des modules microdifférentiels. Dans la suite, nous aurons à nouveau besoin de ces théorèmes que nous transcrivons ci-dessous pour les opérateurs de $\mathcal{D}_{X \mid Y}\{r\}$ en deux lemmes.

LEMME 1.3.4. Si $Z=\left\{(x, t) \in X \mid x_{1}=0\right\}$ est non $\{r\}$-microcaractéristique pour le module $\mathcal{D}_{X} / \mathcal{D}_{X} P$, on a un isomorphisme:

$$
\mathcal{D}_{X \mid Y}\{r\} / \mathcal{D}_{X \mid Y}\{r\} P={ }^{\mathcal{D}_{X \mid Y}\{r\}} / \mathcal{D}_{X \mid Y}\{r\} Q
$$

où $Q$ est un opérateur de $\mathcal{D}_{X \mid Y}\{r\}$ de type Weierstrass en $D_{x_{1}}$ c'est-à-dire de la forme:

$$
Q\left(x, t, D_{x}, D_{t}\right)=D_{x_{1}}^{m}+\sum_{k=0}^{m-1} Q_{k}\left(x, t, D_{x^{\prime \prime}}, D_{t}\right) D_{x_{1}}^{k} .
$$

Dans cette formule, l'opérateur $Q_{k}$ ne dépend pas de $D_{x_{1}}$ (i.e. commute avec $x_{1}$ ) et l'ordre de $Q_{k}\left(x, t, D_{x^{\prime \prime}}, D_{t}\right) D_{x_{1}}^{k}$ pour la $F_{r}$-filtration est inférieur ou égal à celui de $D_{x_{1}}^{m}$.

Si $r_{0}$ est la première pente de $P$ supérieure à $r$, (donc $\sigma_{s}(P)$ est indépendant de $s \in] r, r_{0}\left[\right.$ ), alors pour tout $s \in\left[r, r_{0}\left[\right.\right.$, tout opérateur $S$ de $\mathcal{D}_{X \mid Y}(s)$ s'écrit de manière unique $S=U P+R$ avec

$$
R\left(x, t, D_{x}, D_{t}\right)=\sum_{k=0}^{m-1} R_{k}\left(x, t, D_{x^{\prime \prime}}, D_{t}\right) D_{x_{1}}^{k}
$$


et l'ordre de UP et de chaque $R_{k} D_{x_{1}}^{k}$ pour la $F_{r^{\prime}}$-filtration $\left(r^{\prime} \in[r, s]\right)$ est inférieur ou égal à celui de $S$.

LEMME 1.3.5 On suppose que $Z=\left\{(x, t) \in X \mid x_{1}=0\right\}$ est non (r)-microcaractéristique pour le module $\mathcal{D}_{X} / \mathcal{D}_{X} P$ et on note $r_{1}$ est la première pente de $P$ inférieure $\grave{a} r, \quad\left(\right.$ donc $\sigma_{s}(P)$ est indépendant de $\left.s \in\right] r_{1}, r[)$. Alors, pour tout $s \in\left[r_{1}, r[\right.$, on a un isomorphisme:

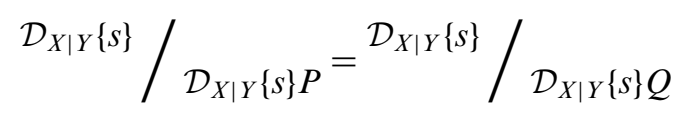

où $Q$ est un opérateur de $\mathcal{D}_{X \mid Y}\{s\}$ de type Weierstrass en $D_{x_{1}}$ c'est-à-dire de la forme:

$$
Q\left(x, t, D_{x}, D_{t}\right)=D_{x_{1}}^{m}+\sum_{k=0}^{m-1} Q_{k}\left(x, t, D_{x^{\prime \prime}}, D_{t}\right) D_{x_{1}}^{k}
$$

Dans cette formule, l'opérateur $Q_{k}$ ne dépend pas de $D_{x_{1}}$ (i.e. commute avec $x_{1}$ ) et l'ordre de $Q_{k}\left(x, t, D_{x^{\prime}}, D_{t}\right) D_{x_{1}}^{k}$ pour la $F_{s}$-filtration est inférieur ou égal à celui de $D_{x_{1}}^{m}$.

Pour tout $s \in\left[r_{1}, r\left[\right.\right.$, tout opérateur $S$ de $\mathcal{D}_{X \mid Y}(s)$ s'écrit de manière unique $S=U P+R$ avec:

$$
R\left(x, t, D_{x}, D_{t}\right)=\sum_{k=0}^{m-1} R_{k}\left(x, t, D_{x^{\prime \prime}}, D_{t}\right) D_{x_{1}}^{k}
$$

et l'ordre de UP et de chaque $R_{k} D_{x_{1}}^{k}$ pour la $F_{r^{\prime}}$-filtration $\left(r^{\prime} \in[s, r]\right)$ est inférieur ou égal à celui de $S$.

Démonstration. Ces deux lemmes sont des conséquences directes des théorèmes 2.7.1 et 2.7.2 de [5]. Il suffit de remarquer que si $P$ n'a pas de pentes dans l'intervalle $] r, r_{0}\left[\right.$, si $r<s<r^{\prime}<r_{0}$, la fonction $\sigma_{s}(P)$ que nous avons définie dans le paragraphe 1.1 est égale à la fonction $\sigma\left(r^{\prime}, r\right)(P)$ définie dans [5]. Si $Z$ est non $\{r\}$-microcaractéristique pour $P$, cette fonction vérifie les hypothèses du théorème de préparation de Weierstrass en $x_{1}^{*}$ et les deux théorèmes précités s'appliquent. Il reste à remarquer que, par définition, on a $\mathcal{D}_{X \mid Y}\{r\}=\left.\mathcal{D}_{\Lambda}^{2}(r, r)\right|_{Y}$ pour obtenir le premier lemme.

Le deuxième lemme est analogue mais une hypothèse du type 'non $(r)$-microcaractéristique' induit des résultats sur la fonction $\sigma(r, s)(P)$ pour $s>r$ donc un théorème de préparation dans $\mathcal{D}_{\Lambda}^{2}(r, s)$ c'est-à-dire dans $\mathcal{D}_{X \mid Y}\{s\}$ et non dans $\mathcal{D}_{X \mid Y}\{r\}$.

\subsection{PROBLÈME DE CAUCHY}

De même que le module $\varphi^{*} \mathcal{M}$ sert à définir le problème de Cauchy pour les fonctions holomorphes, le faisceau $\varphi^{*} \mathcal{M}\{r\}$ définit un problème de Cauchy pour les faisceaux 
$\mathcal{O}_{X \mid Y}\{r\}$ (complété formel de $\mathcal{O}_{X}$ le long de $Y$ à croissance Gevrey) et les faisceaux de cohomologie $\mathcal{B}_{Y \mid X}\{r\}$.

Supposons tout d'abord que $Y$ est une hypersurface et notons $j$ le plongement $X-Y \hookrightarrow X$. Le faisceau des hyperfonctions holomorphes de [12] est le faisceau de cohomologie de $\mathcal{O}_{X}$ à support dans $Y$, c'est-à-dire $\mathcal{B}_{Y \mid X}^{\infty}=\mathcal{H}_{Y}^{1}\left(\mathcal{O}_{X}\right)=$ $j_{*} j^{-1} \mathcal{O}_{X} / \mathcal{O}_{X}$, quotient du faisceau des fonctions ayant une singularité sur $Y$ par celui des fonctions holomorphes. Si on remplace les fonctions à singularités essentielles par le faisceau $\mathcal{O}_{X[* Y]}$ des fonctions méromorphes à pôles sur $Y$ on obtient le faisceau de cohomologie algébrique $\mathcal{B}_{Y \mid X}=\mathcal{H}_{[Y]}^{1}\left(\mathcal{O}_{X}\right)=\mathcal{O}_{X[* Y] / \mathcal{O}_{X}}$.

Pour tout réel $r>1$, on désigne ([6, 9]) par $\mathcal{B}_{Y \mid X}(r)$ le sous-faisceau de $\mathcal{B}_{Y \mid X}^{\infty}$ image des fonctions de $j_{*} j^{-1} \mathcal{O}_{X}$ qui sont majorées par une fonction $C_{0} \exp \left(C|t| \frac{-1}{r-1}\right)$ où $C$ et $C_{0}$ sont des constantes positives et $t$ une équation de $Y$. De même, $\mathcal{B}_{Y \mid X}\{r\}$ est l'image des fonctions qui, pour tout $\varepsilon>0$, sont majorées par une fonction $C_{\varepsilon} \exp \left(\varepsilon|t| \frac{-1}{r-1}\right)$.

Lorsque $Y$ est une sous-variété de $X$ de codimension $d$, on pose encore, suivant [12], $\mathcal{B}_{Y \mid X}^{\infty}=\mathcal{H}_{Y}^{d}\left(\mathcal{O}_{X}\right)$ et $\mathcal{B}_{Y \mid X}=\mathcal{H}_{[Y]}^{d}\left(\mathcal{O}_{X}\right)$. Si $Y$ est l'intersection de $d$ hypersurfaces $T_{i}$ d'équations $\left\{t_{i}=0\right\}, \mathcal{B}_{X \mid Y}^{\infty}$ est le quotient du faisceau des fonctions holomorphes définies en dehors de la réunion des hypersurfaces $T_{i}$ par celui des fonctions holomorphes en dehors d'une réunion de $d-1$ hypersurfaces $T_{i}$. Le faisceau $\mathcal{B}_{Y \mid X}$ est donné par les fonctions méromorphes et les faisceaux $\mathcal{B}_{Y \mid X}(r)$ et $\mathcal{B}_{Y \mid X}\{r\}$ par les fonctions à croissance de type $C_{0} \exp \left(C\left|t_{1} \cdots t_{d}\right|_{r-1}\right)$ ou $C_{\varepsilon} \exp \left(\varepsilon\left|t_{1} \cdots t_{d}\right|_{r-1}^{-1}\right)$.

Dans un système de coordonnées locales $\left(x_{1}, \ldots, x_{p}, t_{1}, \ldots, t_{d}\right)$ de $X$ pour lequel $Y=\{(x, t) \in X \mid t=0\}$, un élément de $\mathcal{B}_{Y \mid X}^{\infty}$ est représenté de manière unique par un développement de Laurent:

$$
f(x, t)=\sum_{\alpha_{1}<0, \ldots, \alpha_{d}<0} a_{\alpha}(x) t^{\alpha}
$$

Les éléments de $\mathcal{B}_{Y \mid X}$ correspondent aux sommes finies et les éléments de $\mathcal{B}_{Y \mid X}(r)$ définis sur un ouvert $U$ de $Y$ sont ceux qui vérifient:

$$
\forall K \Subset U, \exists C_{0}>0, C_{1}>0, \forall x \in K, \forall \alpha, \quad\left|a_{\alpha}(x)\right|<C_{0} C_{1}^{|\alpha|} \frac{1}{(|\alpha| !)^{r-1}}
$$

tandis que pour $\mathcal{B}_{Y \mid X}\{r\}$ la majoration devient

$$
\forall K \Subset U, \forall \varepsilon>0, \exists C_{\varepsilon}>0, \forall x \in K, \forall \alpha, \quad\left|a_{\alpha}(x)\right|<C_{\varepsilon} \varepsilon^{|\alpha|} \frac{1}{(|\alpha| !)^{r-1}}
$$

On note encore $\mathcal{B}_{Y \mid X}^{\infty}=\mathcal{B}_{Y \mid X}$ et $\mathcal{B}_{Y \mid X}\{1\}=\mathcal{B}_{Y \mid X}^{\infty}$. On obtient ainsi une famille de $\mathcal{D}_{X}$-modules décroissante en $r \geqslant 1$ :

$$
\mathcal{B}_{Y \mid X} \subset \mathcal{B}_{Y \mid X}\{r\} \subset \mathcal{B}_{Y \mid X}(r) \subset \mathcal{B}_{Y \mid X}^{\infty}
$$


On peut vérifier par un calcul direct que $\mathcal{B}_{Y \mid X}\{r\}$ est un $\mathcal{D}_{X \mid Y}\{s\}$-module pour $s \leqslant r$ et que $\mathcal{B}_{Y \mid X}(r)$ est un $\mathcal{D}_{X \mid Y}\{s\}$-module pour $s<r$. Le résultat suivant a été démontré dans [5, Corollaire 3.2.4]:

PROPOSITION 1.4.1. Si $\varphi$ est non $\{r\}$-microcaractéristique pour $\mathcal{M}$, on a un isomorphisme canonique:

$$
\varphi^{-1} \mathbb{R} \mathcal{H} \operatorname{om}_{\mathcal{D}_{X}}\left(\mathcal{M}, \mathcal{B}_{Y \mid X}\{r\}\right) \stackrel{\sim}{\longrightarrow} \mathbb{R} \mathcal{H} \operatorname{om}_{\mathcal{D}_{Z \mid Y^{\prime}}\{r\}}\left(\varphi^{*} \mathcal{M}\{r\}, \mathcal{B}_{Y^{\prime} \mid Z}\{r\}\right)
$$

et si $\varphi$ est non (r)-microcaractéristique pour $\mathcal{M}$, on a un isomorphisme canonique:

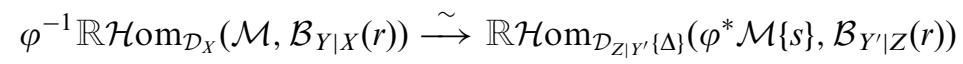

pour $s<r$ assez proche de $r$.

Le point de vue dual consiste à regarder le complété formel du faisceau des fonctions holomorphes le long de $Y$ que l'on notera $\mathcal{O}_{X \mid \hat{Y}}$. Dans les coordonnées locales $(x, t)$, une section de $\mathcal{O}_{X \hat{\mid} Y}$ sur un ouvert $U$ de $Y$ est une série formelle:

$$
f(x, t)=\sum_{\alpha \in \mathbb{N}^{d}} a_{\alpha}(x) t^{\alpha}
$$

de fonctions $a_{\alpha}(x)$ holomorphes sur $U$.

On définit alors $\mathcal{O}_{X \mid Y}\{r\}$ comme le sous-faisceau des séries qui satisfont:

$$
\forall K \Subset U, \exists C_{0}>0, C_{1}>0, \forall x \in K, \forall \alpha, \quad\left|a_{\alpha}(x)\right|<C_{0} C_{1}^{|\alpha|}(|\alpha| !)^{r-1}
$$

Il est immédiat que $\mathcal{O}_{X \mid Y}\{r\}$ est le quotient de $\mathcal{D}_{X \mid Y}\{r\}$ par l'idéal engendré par les champs de vecteurs, c'est-à-dire:

$$
\mathcal{O}_{X \mid Y}\{r\}=\left.\mathcal{D}_{X \mid Y}\{r\} \otimes_{\mathcal{D}_{X} \mid Y} \mathcal{O}_{X}\right|_{Y}
$$

Comme dans le cas de la cohomologie, le problème de Cauchy est bien posé en utilisant les images inverses de type $(r)$ :

PROPOSITION 1.4.2. Si $\varphi$ est non $\{r\}$-microcaractéristique pour $\mathcal{M}$, on a un isomorphisme canonique:

$$
\varphi^{-1} \mathbb{R} \mathcal{H} \operatorname{om}_{\mathcal{D}_{X}}\left(\mathcal{M}, \mathcal{O}_{X \mid Y}\{r\}\right) \stackrel{\sim}{\longrightarrow} \mathbb{R} \mathcal{H} \operatorname{om}_{\mathcal{D}_{Z \mid Y^{\prime}}\{r\}}\left(\varphi^{*} \mathcal{M}\{r\}, \mathcal{O}_{Z \mid Y^{\prime}}\{r\}\right)
$$

Démonstration. Ce résultat pourrait sans doute être obtenu par dualité à partir du précédent, il est plus simple de le démontrer directement. La première étape consiste à se ramener au cas d'une hypersurface $Z$ de $X$ et d'un module de la forme $\mathcal{D}_{X} / \mathcal{D}_{X} P$. La méthode est standard, voir par exemple [3, th 2.4.6] ou [2, th 2.7.4].

Dans le cas d'un seul opérateur, on peut utiliser la méthode de [13, th 3.1.1]. Les deux ingrédients sont un théorème de division, le lemme 1.3.4, et un théorème de Cauchy abstrait [13, th 3.2.1]. La norme formelle de Boutet de Monvel doit être 
remplacée par la norme $N_{(r, s)}$ définie en [5, Déf 2.4.3] et qui est adaptée à $\mathcal{D}_{X \mid Y}\{r\}$. Comme cette norme est assez compliquée, on peut aussi montrer par un calcul direct que les opérateurs de $\mathcal{D}_{X \mid Y}\{r\}$ opèrent continûment sur $\mathcal{O}_{X \mid Y}\{r\}$.

Dans les propositions 1.4.1 et 1.4.2, on utilise l'image inverse $\varphi^{*} \mathcal{M}\{r\}$ qui dépend de $r$ et dans la suite nous allons nous efforcer d'éliminer cette dépendance et nous montrerons que sous certaines conditions on peut remplacer ce module par l'image inverse usuelle $\varphi^{*} \mathcal{M}$. Il faut remarquer cependant que en général le module $\varphi^{*} \mathcal{M}\{r\}$ dépend effectivement de $r$.

\section{Image inverse et pentes d'un $\mathcal{D}$-module}

\subsection{LE CAS D'UN MODULE COHÉRENT QUELCONQUE}

On reprend les notations précédentes, c'est-à-dire que l'on considère une sous-variété lisse $Y$ de $X$ et une application $\varphi: Z \rightarrow X$ transverse à $Y$. On note $Y^{\prime}=\varphi^{-1}(Y)$.

Étant donnés deux indices $r<s$, on a toujours un morphisme canonique de $\mathcal{D}_{Z \mid Y^{\prime}}\{s\}$-modules:

$$
\mathcal{D}_{Z \mid Y^{\prime}}\{s\} \otimes_{\mathcal{D}_{Z \mid Y^{\prime}}\{r\}} \varphi^{*} \mathcal{M}\{r\} \longrightarrow \varphi^{*} \mathcal{M}\{s\}
$$

On dira que $\varphi^{*} \mathcal{M}\{r\}$ 'ne dépend pas de $r \in[a, b]$ ' si ce morphisme est un isomorphisme pour $r$ et $s$ dans l'intervalle $[a, b]$. C'est un abus de langage justifié par le fait que dans ce cas $\varphi^{*} \mathcal{M}\{r\}$ et $\varphi^{*} \mathcal{M}\{s\}$ ont mêmes solutions dans un $\mathcal{D}_{Z \mid Y^{\prime}}\{s\}$-module et mêmes cycles microcaractéristiques (lemme 1.2.1).

Le premier résultat est que l'indépendance de $r$ est satisfaite tant que l'on ne franchit pas de pente. Cela implique en particulier que le nombre de sauts est fini (localement sur $Y$ ). Auparavant, nous allons montrer deux lemmes qui permettront de se réduire au cas d'un seul opérateur.

LEMME 2.1.1. Si $\varphi$ est un morphisme lisse, alors le morphisme (2.1.1) est un isomorphisme pour tout $(r, s)$.

Démonstration. Il suffit de montrer le résultat pour $\mathcal{M}=\mathcal{D}_{X}$. D'après [5, lemme 2.10.2], si $\varphi$ est lisse, $\varphi^{*} \mathcal{D}_{X \mid Y}\{r\}$ est cohérent sur $\mathcal{D}_{Z \mid Y^{\prime}}\{r\}$ pour tout $r$ et on a:

$$
\varphi^{*} \mathcal{D}_{X \mid Y}\{r\}=\mathcal{D}_{Z \mid Y^{\prime}}\{r\} \otimes_{\mathcal{D}_{Z}} \mathcal{D}_{Z \rightarrow X}
$$

d'où le résultat.

LEMME 2.1.2. Soit $Z$ une hypersurface de $X$ non $\{r\}$-microcaractéristique pour le $\mathcal{D}_{X}$-module cohérent $\mathcal{M}$, il existe (localement) un morphisme surjectif $\mathcal{L} \rightarrow \mathcal{M}$ 
où $\mathcal{L}$ est une somme directe:

$$
\mathcal{L}=\bigoplus_{i=1}^{N} \mathcal{D}_{X} / \mathcal{D}_{X} P_{i}
$$

telle que $Z$ est non $\{r\}$-microcaractéristique pour chaque $\mathcal{D}_{X} / \mathcal{D}_{X} P_{i}$.

Démonstration. La démonstration est la même que dans le cas des variétés caractéristiques (cf [2] par exemple):

Puisque $Z$ est une hypersurface, le fibré $T_{\Lambda^{\prime}}^{*} \Lambda$ qui intervient dans la définition 1.3.1 est un fibré en droite et par homogénéité pour que $Z$ soit non $\{r\}$-microcaractéristique pour $\mathcal{M}$ il suffit qu'un point non nul de cette droite ne se trouve pas dans $C h_{\Lambda}\{r\}(\mathcal{M})$. Si $u$ est une section de $\mathcal{M}$, la variété $\{r\}$-microcaractéristique de $\mathcal{D}_{X} u$ est contenue dans celle de $\mathcal{M}$ donc $Z$ est non $\{r\}$-microcaractéristique pour $\mathcal{D}_{X} u$. Comme la variété $\{r\}$-microcaractéristique de $\mathcal{D}_{X} u$ est égale à l'intersection des variétés microcaractéristiques des opérateurs qui annulent $u$, il existe un opérateur $P$ qui annule $u$ et qui n'est pas $\{r\}$-microcaractéristique.

On obtient le lemme en considérant localement une famille finie $u_{1}, \ldots, u_{N}$ de générateurs de $\mathcal{M}$ et pour chaque $i$ un opérateur $P_{i}$ qui annule $u_{i}$ et n'est pas $\{r\}$-microcaractéristique.

PROPOSITION 2.1.3. Si $\mathcal{M}$ n'a pas de pentes (le long de $Y$ ) dans l'intervalle $] r_{0}, r_{1}[$ et si $\varphi$ est non $\left\{r_{0}\right\}$-microcaractéristique pour $\mathcal{M}$, alors $\varphi^{*} \mathcal{M}\{r\}$ 'ne dépend pas de $r \in\left[r_{0}, r_{1}[\right.$ '.

Démonstration. Le résultat étant stable par composition des morphismes, on peut décomposer $\varphi$ en une immersion de $Z$ dans le graphe de $\varphi$ suivie de la projection du graphe sur $X$. Le cas de la projection étant démontré par le lemme 2.1.1, il suffit d'étudier le cas d'une immersion que l'on peut décomposer en une suite d'immersions de variété de codimension 1 . Il suffit donc de traiter le cas où $Z$ est une hypersurface lisse de $X$.

Sous les hypothèses de la proposition, la variété $C h_{\Lambda}\{r\}(\mathcal{M})$ ne dépend pas de $r \in\left[r_{0}, r_{1}[\right.$ donc $Z$ est non $\{r\}$-microcaractéristique pour tout $r$ de cet intervalle. Pour montrer la proposition, il suffit donc de montrer qu'il existe $\varepsilon>0$ tel que $\varphi^{*} \mathcal{M}\{r\}$ ne dépend pas de $r \in\left[r_{0}, r_{0}+\varepsilon[\right.$.

D'après le lemme 1.3.4, si $\mathcal{M}$ est de la forme $\mathcal{D}_{X} / \mathcal{D}_{X} P$, le module $\varphi^{*} \mathcal{M}\{r\}$ est isomorphe à $\left(\mathcal{D}_{Z \mid Y^{\prime}}\{r\}\right)^{N}$ pour tout $r$ dans un intervalle $\left[r_{0}, r_{0}+\varepsilon[\right.$. La proposition est donc vérifiée dans ce cas et on obtient le cas général en utilisant le lemme 2.1.2.

Le deuxième résultat général que l'on peut obtenir est la surjectivité:

PROPOSITION 2.1.4. Si $Z$ est non $\{$ s\}-microcaractéristique pour $\mathcal{M}$, alors le morphisme 2.1.1. est surjectif.

Démonstration. Comme précédemment, on se ramène au cas d'un seul opérateur et d'une hypersurface en utilisant les lemmes 2.1.1 et 2.1.2. On peut aussi fixer des 
coordonnées locales dans lesquelles $Z=\left\{x_{1}=0\right\}$. Alors, si $\mathcal{M}=\mathcal{D}_{X} / \mathcal{D}_{X} P$, le module $\varphi^{*} \mathcal{M}\{r\}$ est, par définition, égal à:

$$
\varphi^{*} \mathcal{M}\{r\}={ }^{\mathcal{D}_{X \mid Y}\{r\}} /\left(\mathcal{D}_{X \mid Y}\{r\} P+x_{1} \mathcal{D}_{X \mid Y}\{r\}\right)
$$

Notons pour simplifier (avec ici $Y^{\prime}=Y \cap Z$ ):

$$
\widetilde{\mathcal{D}}=\mathcal{D}_{Z \mid Y^{\prime}}\{s\} \otimes_{\mathcal{D}_{Z \mid Y^{\prime}}\{r\}}\left(\left.\mathcal{D}_{X \mid Y}\{r\}\right|_{Y^{\prime}}\right)
$$

On a donc

$$
\mathcal{D}_{Z \mid Y^{\prime}}\{s\} \otimes_{\mathcal{D}_{Z \mid Y^{\prime}}\{r\}} \varphi^{*} \mathcal{M}\{r\}=\widetilde{\mathcal{D}} /\left(\widetilde{\mathcal{D}} P+x_{1} \widetilde{\mathcal{D}}\right)
$$

et le morphisme 2.1.1 est alors égal à:

$$
\widetilde{\mathcal{D}} /\left(\widetilde{\mathcal{D}} P+x_{1} \widetilde{\mathcal{D}}\right) \rightarrow^{\mathcal{D}_{X \mid Y}\{s\}} /{ }_{\left(\mathcal{D}_{X \mid Y}\{s\} P+x_{1} \mathcal{D}_{X \mid Y}\{s\}\right)}
$$

Le théorème de division (lemme 1.3.4) montre que tout opérateur de $\mathcal{D}_{X \mid Y}\{s\}$ s'écrit comme la somme d'un opérateur de $\mathcal{D}_{X \mid Y}\{s\} P$, d'un opérateur de $x_{1} \mathcal{D}_{X \mid Y}\{s\}$ et d'un polynôme en $D_{x_{1}}$ à coefficients dans $\mathcal{D}_{Z \mid Y^{\prime}}\{s\}$ c'est-à-dire précisément d'un opérateur de $\widetilde{\mathcal{D}}$. Ceci montre que le morphisme est bien surjectif.

Si $\varphi$ est non $(r)$-microcaractéristique pour $\mathcal{M}$, le cycle microcaractéristique du module image inverse se calcule à partir du cycle correspondant de $\mathcal{M}$ :

THÉORÈME 2.1.5. (1) Si $\varphi$ est non $\{r\}$-microcaractéristique pour $\mathcal{M}$ on a:

$$
\widetilde{\Sigma}_{\Lambda^{\prime}}^{0}(r)\left(\varphi^{*} \mathcal{M}\{r\}\right)=\varrho \varpi^{-1} \widetilde{\Sigma}_{\Lambda}^{0}(r)(\mathcal{M})
$$

(2) Si e est non (r)-microcaractéristique pour $\mathcal{M}$ le long de $Y$ et si $\mathcal{M}$ n'a pas de pentes dans l'intervalle $[s, r[$, on a:

$$
\widetilde{\Sigma}_{\Lambda^{\prime}}(r)\left(\varphi^{*} \mathcal{M}\{s\}\right)=\varrho \varpi^{-1} \widetilde{\Sigma}_{\Lambda}(r)(\mathcal{M})
$$

Démonstration. La démonstration est essentiellement la même que pour la formule donnant le cycle caractéristique de la restriction d'un $\mathcal{D}_{X}$-module. On peut recopier par exemple la démonstration de [13, th $3.4 .2 \mathrm{ch}$ II] et nous ne donnerons ici que les principales étapes de la démonstration. Nous considérons tout d'abord la première partie du théorème.

(a) Comme dans la démonstration de la proposition 2.1.3, on se ramène au cas où $\varphi$ est l'immersion d'une hypersurface $Z$ dans $X$. On peut alors choisir des coordonnées dans lesquelles $Z$ est l'hypersurface $x_{1}=0$.

(b) D'après le lemme 2.1.2, toute section $u$ de $\mathcal{M}$ est annulée par un opérateur $P$ tel que $Z$ est non $\{r\}$-microcaractéristique pour $P$. 
(c) Soit $\widetilde{\mathcal{D}_{X \mid Y}}\{r\}$ le sous-faisceau de $\mathcal{D}_{X \mid Y}\{r\}$ des opérateurs indépendants de $D_{X_{1}}$. Le théorème de division (lemme 1.3.4) montre que pour toute section $u$ de $\mathcal{M}$, il existe un entier $m$ tel que:

$$
F_{r}^{k} \mathcal{D}_{X \mid Y}\{r\} u \subset \sum_{i=0}^{m-1} F_{r}^{k-i} \widetilde{\mathcal{D}_{X \mid Y}}\{r\} D_{x_{1}}^{i} u
$$

et donc, si $u_{1}, \ldots, u_{q}$ engendrent localement $\mathcal{M}$, celui-ci admet une bonne $F_{r}$-filtration de la forme:

$$
\mathcal{M}_{k}=\sum_{j=1}^{q} \sum_{i=0}^{m_{j}-1} F_{r}^{k-i} \widetilde{\mathcal{D}_{X \mid Y}}\{r\} D_{x_{1}}^{i} u_{j}
$$

(d) Soit $\mathcal{N}_{k}=\mathcal{M}_{k} /\left(x_{1} \mathcal{M} \cap \mathcal{M}_{k}\right)$. Les faisceaux $\mathcal{N}_{k}$ définissent une bonne filtration de $\mathcal{N}=\mathcal{M} / x_{1} \mathcal{M}$ et donc les faisceaux $\varrho_{*} \mathcal{N}_{k}$ définissent une bonne filtration de $\varrho_{*} \mathcal{N}=\varphi^{*} \mathcal{M}\{r\}$.

(e) On en déduit alors, toujours suivant [13], que le module $\varphi^{*} \mathcal{M}\{r\}$ est cohérent (ce qui était déjà connu) et que le cycle analytique défini par le gradué de $F_{r} \varphi^{*} \mathcal{M}\{r\}$ est l'image par $\varrho \varpi^{-1}$ du gradué de $F_{r} \mathcal{M}$. Ces cycles sont, par définitions les cycles $\widetilde{\boldsymbol{\Sigma}}_{\Lambda^{\prime}}^{0}(r)\left(\varphi^{*} \mathcal{M}\{r\}\right)$ et $\varrho \varpi^{-1} \widetilde{\boldsymbol{\Sigma}}_{\Lambda}^{0}(r)(\mathcal{M})$ d'où le théorème (cas 1$)$.

(f) La démonstration de la deuxième partie est analogue. On remarque tout d'abord que grâce à la proposition 2.1.3, on peut localiser la démonstration en $s$, c'est-à-dire qu'il suffit de montrer le résultat sur les intervalles $\left[s_{i}, s_{i+1}\right.$ [ d'une partition finie de $[s, r[$. On remarque alors comme en (b) que toute section $u$ de $\mathcal{M}$ est annulée par un opérateur $P$ tel que $Z$ est non $(r)$-microcaractéristique pour $P$ et de plus $P$ n'a pas de pentes sur un intervalle $\left[s^{\prime}, r[\right.$. Prenant un nombre fini de générateurs locaux de $\mathcal{M}$ annulés par des opérateurs du type précédent sans pentes dans un intervalle commun $\left[s_{0}, r\right.$, on peut comme en (c) définir une bonne filtration de $\mathcal{M}$ de la forme:

$$
\mathcal{M}_{k}=\sum_{j=1}^{q} \sum_{i=0}^{m_{j}-1} F_{r}^{k-i} \widetilde{\mathcal{D}_{X \mid Y}}\left\{s_{0}\right\} D_{x_{1}}^{i} u_{j}
$$

(Le lemme 1.3.5 assure que le théorème de division sur $\mathcal{D}_{X \mid Y}\left\{s_{0}\right\}$ respecte la $F_{r}$-filtration). La suite de la démonstration est la même que précédemment et on obtient le résultat sur un intervalle $\left[s_{0}, r\left[\right.\right.$. On peut alors reprendre à partir de $s_{0}$ mais il faut s'assurer que l'on atteint $s$ par un nombre fini de segments. Pour cela on applique la proposition 3.4.2. de [6].

COROLLAIRE 2.1.6. Si $\varphi$ est non $\{r\}$-microcaractéristique pour $\mathcal{M}$ on a:

$$
\widetilde{C h}_{\Lambda^{\prime}}\{r\}\left(\varphi^{*} \mathcal{M}\{r\}\right)=\varrho \varpi^{-1} \widetilde{C h}_{\Lambda}\{r\}(\mathcal{M})
$$


et si $\varphi$ est non (r)-microcaractéristique pour $\mathcal{M}$ on a pour \& assez petit:

$$
\widetilde{C} h_{\Lambda^{\prime}}(r)\left(\varphi^{*} \mathcal{M}\{r-\varepsilon\}\right)=\varrho \varpi^{-1} \widetilde{C} h_{\Lambda}(r)(\mathcal{M})
$$

(Le résultat était déjà connu dans le cas des variétés microcaractéristiques [5], nous l'étendons ici au cas des cycles.)

Démonstration. Si $\varphi$ est non $\{r\}$-microcaractéristique on peut appliquer la partie (1) du théorème 2.1.5 qui donne directement le résultat puisque $\widetilde{C} h_{\Lambda}\{r\}(\mathcal{M})$ ne dépend que de $\widetilde{\Sigma}_{\Lambda}^{0}(r)(\mathcal{M})$.

Si $\varphi$ est non $(r)$-microcaractéristique, on choisit un $\varepsilon$ assez petit pour que $\mathcal{M}$ n'ait pas de pentes sur l'intervalle $[r-\varepsilon, r$ [ et on applique la partie (2) du théorème 2.1.5.

COROLLAIRE 2.1.7. Si $\left\{r_{0}\right\}$ n'est pas une pente de $\mathcal{M}$ et si $Z$ est non $\left\{r_{0}\right\}$-microcaractéristique pour $\mathcal{M}, \varphi^{*} \mathcal{M}\{r\}$ ne dépend pas de $r$ au voisinage de $r_{0}$ et $r_{0} n^{\prime} e s t$ pas une pente de $\varphi^{*} \mathcal{M}\{r\}$.

En particulier, si le module $\mathcal{M}$ n'a aucune pente et si $Z$ est non microcaractéristique pour tout $r$, le module induit usuel $\varphi^{*} \mathcal{M}$ n'a aucune pente.

Démonstration. Le module $\varphi^{*} \mathcal{M}\{r\}$ ne dépend pas de $r$ d'après la proposition 2.1.3 et d'après le théorème 2.1.5 la variété $\{r\}$-microcaractéristique de ce module est égale à $\varrho \varpi^{-1} \widetilde{\Sigma}_{\Lambda}(r)(\mathcal{M})$ qui est bihomogène. Elle est donc elle-même bihomogène et $r_{0}$ n'est pas une pente de $\varphi^{*} \mathcal{M}\{r\}$.

\subsection{LE CAS D'UN MODULE HOLONOME}

Le premier résultat que l'on obtient dans le cas holonome est que l'indépendance de $r$ du module induit peut se lire sur les cycles microcaractéristiques:

LEMME 2.2.1. Soit $\mathcal{M}$ un $\mathcal{D}_{X}$-module holonome, $r$ et $s$ deux nombres verifiant $s>r \geqslant 1$ et tels que $\varphi$ soit non $\{s\}$ et non $\{r\}$-microcaractéristique. On suppose que:

$$
\widetilde{\Sigma}_{\Lambda^{\prime}}^{0}\left(\varphi^{*} \mathcal{M}\{s\}\right)=\left.\widetilde{\Sigma}_{\Lambda^{\prime}}\left(\varphi^{*} \mathcal{M}\{r\}\right)\right|_{Y}
$$

alors le morphisme 2.1.1 est un isomorphisme.

(Comme dans le lemme 1.2.1, il s'agit d'une égalité entre les germes sur $Y$ car le premier terme est seulement défini sur un voisinage de $Y$ ).

Démonstration. Montrons tout d'abord que $\Sigma_{\Lambda^{\prime}}^{0}(s)\left(\varphi^{*} \mathcal{M}\{s\}\right)$ est (un germe de variété) lagrangienne de $T^{*} \Lambda^{\prime}$.

En effet, si $Z$ est non $\{s\}$-microcaractéristique, l'application $\varrho$ est finie sur la variété $\varpi^{-1} C h_{\Lambda}\{s\}(\mathcal{M})$. Comme $C h_{\Lambda}\{s\}(\mathcal{M})$ est involutive, cela implique qu'aucune de ses composantes n'est contenue dans $\left(T^{*} \Lambda\right) \times_{\Lambda} \Lambda^{\prime}$. (En coordonnées $\left(T^{*} \Lambda\right) \times{ }_{\Lambda} \Lambda^{\prime}$ est la sous-variété de $T^{*} \Lambda$ d'équation $x_{1}=0$ et si une composante irréductible d'une 
variété involutive est contenue dans cette hypersurface, elle est invariante en $x_{1}^{*}$ et la projection correspondante n'est pas finie).

La dimension de $\varrho \varpi^{-1} C h_{\Lambda}\{s\}(\mathcal{M})$ est donc strictement inférieure à la dimension de $C h_{\Lambda}\{s\}(\mathcal{M})$. Comme $\mathcal{M}$ est holonome, $C h_{\Lambda}\{s\}(\mathcal{M})$ est lagrangienne [6, Corollaire 4.1.2], donc de dimension $n=\operatorname{dim}_{\mathbb{C}} X$ et $\varrho \varpi^{-1} C h_{\Lambda}\{s\}(\mathcal{M})$ est de dimension au plus $n-1$. D'après le théorème 2.1.6, $\varrho \varpi^{-1} C h_{\Lambda}\{s\}(\mathcal{M})$ est égale à $C h_{\Lambda^{\prime}}(s)\left(\varphi^{*} \mathcal{M}\{s\}\right)$ qui, par ailleurs est une sous-variété involutive de $T^{*} \Lambda^{\prime}$ donc est lagrangienne.

Enfin, $C h_{\Lambda^{\prime}}(s)\left(\varphi^{*} \mathcal{M}\{s\}\right)$ étant le cône tangent à $\Sigma_{\Lambda}^{\prime 0}(s)\left(\varphi^{*} \mathcal{M}\{s\}\right)$, celle-ci est de même dimension donc est lagrangienne.

Si $Z$ est non $\{s\}$ et non $\{r\}$-microcaractéristique, les modules $\varphi^{*} \mathcal{M}\{s\}$ et $\varphi^{*} \mathcal{M}\{r\}$ sont cohérents et d'après la proposition 2.1 .4 on a un morphisme surjectif $\mathcal{D}_{Z \mid Y^{\prime}}\{s\} \otimes \varphi^{*} \mathcal{M}\{r\} \rightarrow \varphi^{*} \mathcal{M}\{s\}$ dont nous noterons $\mathcal{N}$ le noyau.

On a donc une suite exacte

$$
0 \rightarrow \mathcal{N} \rightarrow \mathcal{D}_{Z \mid Y^{\prime}}\{s\} \otimes \varphi^{*} \mathcal{M}\{r\} \rightarrow \varphi^{*} \mathcal{M}\{s\} \rightarrow 0
$$

dont les deux derniers termes ont même cycle microcaractéristique, donc par additivité la partie de dimension $n-1$ du cycle microcaractéristique de $\mathcal{N}$ est nulle. Comme la variété microcaractéristique de $\mathcal{N}$ est involutive, elle doit être vide et donc $\mathcal{N}$ est nul et le morphisme 2.1.1 est un isomorphisme.

THÉORÈME 2.2.2. Soit $\mathcal{M}$ et un $\mathcal{D}_{X}$-module holonome tel que $\varphi$ est non $\{r\}$-microcaractéristique pour $r$ dans un intervalle $] r_{0}, r_{1}\left[\right.$, alors $\varphi^{*} \mathcal{M}\{r\}$ ne dépend pas de $r$ dans cet intervalle.

Démonstration. Fixons $\left.r_{2} \in\right] r_{0}, r_{1}\left[\right.$. Si $r_{2}$ n'est pas une pente de $\mathcal{M}$ alors $\varphi^{*} \mathcal{M}\{r\}$ ne dépend pas de $r$ au voisinage de $r_{2}$ d'après 2.1.3.

Si $r_{2}$ est une pente, les nombres $r$ proches de $r_{2}$ n'en sont pas (les pentes sont localement en nombre fini). D'après la proposition 2.1.3 $\varphi^{*} \mathcal{M}\{r\}$ ne dépend pas de $r$ sur un intervalle $\left[r_{2}, r_{2}+\varepsilon\right.$ [. Par ailleurs, d'après le théorème 2.1 .5 , on a l'égalité de cycles:

$$
\widetilde{\Sigma}_{\Lambda^{\prime}}^{0}\left(r_{2}\right)\left(\varphi^{*} \mathcal{M}\left\{r_{2}\right\}\right)=\widetilde{\Sigma}_{\Lambda^{\prime}}^{0}\left(r_{2}\right)\left(\varphi^{*} \mathcal{M}\{s\}\right)
$$

pour $s<r_{2}$ assez proche donc d'après le lemme 2.1.5 $\varphi^{*} \mathcal{M}\{r\}$ ne dépend pas de $r$ sur un intervalle $\left[r_{2}-\varepsilon, r_{2}\right]$. Finalement le résultat est vrai sur un voisinage de $r_{2}$.

De ce théorème, nous pouvons déduire le résultat principal de cet article:

COROLLAIRE 2.2.3. Si $\mathcal{M}$ est un $\mathcal{D}_{X}$-module holonome tel que $\varphi$ est non $\{r\}$-microcaractéristique pour tout $r \geqslant 1$, on a:

$$
\forall r \geqslant 1, \quad \varphi^{*} \mathcal{M}(r)=\mathcal{D}_{Z \mid Y^{\prime}}\{r\} \otimes_{\mathcal{D}_{Z}}\left(\varphi^{*} \mathcal{M}\right)
$$

où $\varphi^{*} \mathcal{M}$ désigne l'image inverse usuelle au sens des $\mathcal{D}_{X}$-modules par $\varphi$. 
En appliquant les propositions 1.4 .1 et 1.4 .2 on obtient:

COROLLAIRE 2.2.4. Si $\mathcal{M}$ est un $\mathcal{D}_{X}$-module holonome tel que $\varphi$ est non $\{r\}$-microcaractéristique pour tout $r \geqslant 1$, on a alors des théorèmes de type Cauchy-Kowalevska:

$$
\begin{aligned}
& \left.\mathbb{R} \mathcal{H} \operatorname{lom}_{\mathcal{D}_{X}}\left(\mathcal{M}, \mathcal{B}_{Y \mid X}\{r\}\right)\right|_{Y \cap Z} \stackrel{\sim}{\longrightarrow} \mathbb{R} \mathcal{H} \operatorname{om}_{\mathcal{D}_{Z}}\left(\varphi^{*} \mathcal{M}, \mathcal{B}_{Y^{\prime} \mid Z}\{r\}\right) \\
& \left.\mathbb{R} \mathcal{H} \operatorname{om}_{\mathcal{D}_{X}}\left(\mathcal{M}, \mathcal{B}_{Y \mid X}(r)\right)\right|_{Y \cap Z} \stackrel{\sim}{\longrightarrow} \mathbb{R} \mathcal{H} \operatorname{Hom}_{\mathcal{D}_{Z}}\left(\varphi^{*} \mathcal{M}, \mathcal{B}_{Y^{\prime} \mid Z}(r)\right) \\
& \left.\mathbb{R} \mathcal{H} \operatorname{om}_{\mathcal{D}_{X}}\left(\mathcal{M}, \mathcal{O}_{X \mid Y}\{r\}\right)\right|_{Y \cap Z} \stackrel{\sim}{\longrightarrow} \mathbb{R} \mathcal{H} \operatorname{Hom}_{\mathcal{D}_{Z}}\left(\varphi^{*} \mathcal{M}, \mathcal{O}_{Z \mid Y^{\prime}}\{r\}\right)
\end{aligned}
$$

Le faisceau d'irrégularité de type $r$ est défini $[9,2.4$.] par:

$$
\operatorname{Irr}_{Y}^{*}\{r\}(\mathcal{M})=\mathbb{R} \mathcal{H} \operatorname{om}_{\mathcal{D}_{X}}\left(\mathcal{M}, \mathcal{B}_{Y \mid X}^{\infty} / \mathcal{B}_{Y \mid X}\{r\}\right)
$$

et son dual de Verdier par

$$
\operatorname{Irr}_{Y}\{r\}(\mathcal{M})=\mathbb{R} \mathcal{H} \operatorname{om}_{\mathcal{D}_{X}}\left(\mathcal{M}, \mathcal{O}_{X \mid Y}\{r\} / \mathcal{O}_{X}\right)
$$

Les résultats précédents signifient donc que sous les hypothèses du corollaire 2.2.4 l'image inverse commute à l'irrégularité:

$$
\varphi^{*} \operatorname{Irr}_{Y}\{r\}(\mathcal{M})=\operatorname{Irr}_{Y}\{r\}\left(\varphi^{*} \mathcal{M}\right)
$$

Comme nous l'avons annoncé dans l'introduction, l'intérêt des résultats de ces corollaires tient à ce que les conditions à remplir sont vérifiées de manière générique, plus précisément on a:

PROPOSITION 2.2.5. Si $\mathcal{M}$ est un module holonome et si $Y$ est une hypersurface, il existe une sous-variété lagrangienne homogène $S$ de $T^{*} Y$ telle que une sous-variété $Z$ de $X$ transverse à $Y$ est non $\{r\}$-microcaractéristique pour tout $r$ si et seulement si $Y \cap Z$ est non caractéristique pour $S$, c'est-à-dire si l'intersection de $S$ et du conormal à $Y \cap Z$ dans $Y$ ne rencontre pas $S$.

En particulier, les points de $Y$ par lesquels ne passe aucune hypersurface non $\{r\}$-microcaractéristique pour tout $r$ sont isolés.

Démonstration. Il n'y a qu'un nombre fini de variétés microcaractéristiques distinctes donc la réunion de toutes les variétés microcaractéristiques $C h_{\Lambda}\{r\}(\mathcal{M})$ pour $r \in[1,+\infty]$ est une réunion finie de variétés lagrangiennes bihomogènes donc est elle-même une variété lagrangienne bihomogène $\Sigma$ de $T^{*} \Lambda$. La condition ' $Z$ non $\{r\}$-microcaractéristique pour tout $r \geqslant 1$ ' équivaut à $T_{\Lambda^{\prime}}^{*} \Lambda \cap \Sigma \subset T_{\Lambda}^{*} \Lambda$ et comme nous l'avons remarqué dans [7], paragraphe 1.5, cela équivaut à $Y \cap Z$ non caractéristique pour une sous-variété lagrangienne $S$ de $T^{*} Y$.

D'après la proposition 2.1.7. l'image inverse non microcaractéristique n'augmente pas le nombre de pentes. Pour étudier la réciproque, il faut d'abord remarquer que pour le module $\varphi^{*} \mathcal{M}\{r\}$ on ne sait pas définir la variété $\Sigma_{\Lambda}^{\prime}(r)$ mais seulement un 
germe de variété $\Sigma_{\Lambda^{\prime}}^{0}(r)$ sur $Y$. La condition ' $r$ n'est pas une pente de $\varphi^{*} \mathcal{M}\{s\}$ ' a donc un sens pour $s<r$ mais pas pour $s=r$. Par contre, cette condition ne dépend pas de $s$ dans un intervalle $[r-\varepsilon, r[$ si $\varepsilon$ est assez petit. En effet, pour $\varepsilon$ est assez petit, $\mathcal{M}$ n'a pas de pentes dans l'intervalle $[r-\varepsilon, r$ [ car les pentes sont isolées, et on peut appliquer la proposition 2.1.3.

Considérons une hypersurface $Z$ d'équation locale $f$, notons $Z_{\alpha}$ l'hypersurface $f^{-1}(\alpha)$ et $\varphi_{\alpha}: Z_{\alpha} \rightarrow X$.

PROPOSITION 2.2.6. Soit $\mathcal{M}$ un $\mathcal{D}_{X}$-module holonome et $\left.r \in\right] 1,+\infty[$. On suppose qu'il existe $\varepsilon_{0}>0$ tel que, pour tout $\alpha$ tel que $|\alpha|<\varepsilon_{0}$ et tout $s \in\left[r-\varepsilon_{0}, r[\right.$, l'hypersurface $Z_{\alpha}$ est non (r)-microcaractéristique pour $\mathcal{M}$ et $r$ n'est pas une pente de $\varphi_{\alpha}^{*} \mathcal{M}\{s\}$.

Alors $r$ n'est pas une pente de $\mathcal{M}$.

Démonstration. Fixons tout d'abord $\alpha$ à la valeur 0. D'après le théorème 2.1.5 on a

$$
\Sigma_{\Lambda^{\prime}}(r)\left(\varphi^{*} \mathcal{M}\{s\}\right)=\varrho \varpi^{-1} \Sigma_{\Lambda}(r)(\mathcal{M})
$$

si $s$ est assez proche de $r$. Puisque $r$ n'est pas une pente de $\varphi^{*} \mathcal{M}\{s\}$, la variété involutive $\Sigma_{\Lambda}^{\prime}(r)\left(\varphi^{*} \mathcal{M}\{s\}\right)$ est bihomogène. D'autre part, comme dans la démonstration du lemme 2.2.1, elle est de dimension strictement inférieure à celle de $\Sigma_{\Lambda}(r)(\mathcal{M})$ qui est lagrangienne dans $T^{*} \Lambda$ donc de même dimension que $\Lambda$. La variété $\varrho \varpi^{-1} \Sigma_{\Lambda}(r)(\mathcal{M})$ est donc une sous-variété involutive de $T^{*} \Lambda^{\prime}$ de même dimension que $\Lambda^{\prime}$ donc elle est lagrangienne.

Reprenons les coordonnées et les notations du début du paragraphe 1.3 avec $Z$ d'équation $\left(x_{1}=0\right)$. La variété $\varrho \varpi^{-1} \Sigma_{\Lambda}(r)(\mathcal{M})$ est lagrangienne bihomogène donc d'après [9, prop. 1.1.1.], elle est contenue dans l'hypersurface d'équation $\left\langle\tau, \tau^{*}\right\rangle=0$. Il en est donc de même de $\varpi^{-1} \Sigma_{\Lambda}(r)(\mathcal{M})$ qui est en coordonnées, l'intersection de $\Sigma_{\Lambda}(r)(\mathcal{M})$ avec $x_{1}=0$.

En faisant varier $\alpha$ on coupe $\Sigma_{\Lambda}(r)(\mathcal{M})$ par $x_{1}=\alpha$ et on trouve une variété contenue dans l'hypersurface $\left\langle\tau, \tau^{*}\right\rangle=0$. On voit donc que $\Sigma_{\Lambda}(r)(\mathcal{M})$ est contenue cette hypersurface. Or $\Sigma_{\Lambda}(r)(\mathcal{M})$ est involutive donc invariante par le champ hamiltonien associé à la fonction $\left\langle\tau, \tau^{*}\right\rangle$, c'est-à-dire $\left\langle\tau, \partial / \partial \tau^{*}\right\rangle+\left\langle\tau^{*}, \partial / \partial \tau^{*} \tau\right\rangle$, ce qui signifie exactement que $\Sigma_{\Lambda}(r)(\mathcal{M})$ est homogène pour $H_{\infty}$. Comme elle est déjà $r$-homogène, elle est bihomogène et donc que $r$ n'est pas une pente de $\mathcal{M}$.

Remarque 2.2.7. On peut étendre la proposition en remplaçant l'hypersurface $Z$ par un morphisme $\varphi: Z \rightarrow X$ quelconque et en remplaçant les hypersurfaces $Z_{\alpha}$ par tous les morphismes dont le graphe est voisin de celui de $\varphi$.

En utilisant les résultats de [9] on peut améliorer ce résultat en évitant de faire varier $\varphi$. Pour cela nous utiliserons le cycle d'irrégularité de [9]. A tout cycle lagrangien $r$-homogène $\widetilde{\Sigma}$ de $T^{*} \Lambda$ on associe un cycle $\operatorname{Irr}(\widetilde{\Sigma})$ de $T^{*} Y$ qui est positif si et seulement si $\widetilde{\Sigma}$ l'est et qui est nul si et seulement si $\widetilde{\Sigma}$ est bihomogène. 
Considérons les applications:

$$
T^{*}\left(Y^{\prime}\right) \stackrel{\rho_{0}}{\longleftarrow}\left(T^{*} Y\right) \times_{Y}\left(Y^{\prime}\right) \stackrel{\varpi_{0}}{\longrightarrow} T^{*} Y
$$

Puisque le cycle $\operatorname{Irr}(\widetilde{\Sigma})$ ne dépend que de $\widetilde{\Sigma}$, le théorème 2.1 .5 montre que si $Z$ est non (r)-microcaractéristique pour le module holonome $\mathcal{M}$ et si $\mathcal{M}$ n'a pas de pentes dans l'intervalle $[s, r[$ alors:

$$
\operatorname{Irr}\left(\widetilde{\Sigma}_{\Lambda^{\prime}}(r)\left(\varphi^{*} \mathcal{M}\{s\}\right)\right)=\varrho_{0} \varpi_{0}^{-1} \operatorname{Irr}\left(\widetilde{\Sigma}_{\Lambda}(r)(\mathcal{M})\right)
$$

THÉORÈME 2.2.8. Soit $\mathcal{M}$ un $\mathcal{D}_{X}$-module holonome et $\left.r \in\right] 1,+\infty[$. On suppose que l'hypersurface $Z$ est non (r)-microcaractéristique pour $\mathcal{M}$ et que $r$ n'est pas une pente de $\varphi^{*} \mathcal{M}\{s\}$ pour $s<r$ assez proche de $r$.

Alors $r$ n'est pas une pente de $\mathcal{M}$.

Démonstration. Si $r$ n'est pas une pente de $\varphi^{*} \mathcal{M}\{s\}$, le cycle $\widetilde{\Sigma}_{\Lambda}^{\prime}(r)\left(\varphi^{*} \mathcal{M}\{s\}\right)$ est bihomogène et le cycle d'irrégularité $\left.\operatorname{Irr}\left(\Sigma_{\Lambda^{\prime}}(r)(\varphi)^{*} \mathcal{M}\{r\}\right)\right)$ est nul. D'après l'égalité (2.2.1) il en est de même pour le cycle $\varrho_{0} \varpi_{0}^{-1} \operatorname{Irr}\left(\Sigma_{\Lambda}(r)(\mathcal{M})\right)$.

Si $Z$ est non $(r)$-microcaractéristique, nous avons vu que cela implique que $\varrho$ est propre sur le support de $\Sigma_{\Lambda}(r)(\mathcal{M})$ et donc $\varrho_{0}$ est propre sur le support de $\varpi_{0}^{-1} \operatorname{Irr}\left(\Sigma_{\Lambda}(r)(\mathcal{M})\right)$ donc ce cycle est nul. Le cycle $\operatorname{Irr}\left(\Sigma_{\Lambda}(r)(\mathcal{M})\right)$ est positif et sa restriction par $\varpi_{0}$ a $T^{*} Y \times_{Y}\left(Y^{\prime}\right)$ est nulle donc il est nul au voisinage de $T^{*} Y \times{ }_{Y}\left(Y^{\prime}\right)$ ce qui implique que $r$ n'est pas une pente.

COROLLAIRE 2.2.9. Si $\mathcal{M}$ est un $\mathcal{D}_{X}$-module holonome tel que $Z$ est non $\{r\}$-microcaractéristuqe pour tout $r \geqslant 1$, les pentes de $\mathcal{M}$ au voisinage de $Z$ le long de $Y$ sont les mêmes que celles de $\varphi^{*} \mathcal{M}$ de $Y \cap Z$.

(Il suffit d'appliquer le corollaire 2.2.3 et la proposition précédente).

Le polygone de Newton d'un module holonome $\mathcal{M}$ le long d'une hypersurface $Y$ a été défini dans [7]. C'est la donnée d'un sous-ensemble analytique lagrangien homogène $\mathfrak{S}$ de $T^{*} Y$ et pour chaque composante irréductible de cet ensemble d'un 'polygone', c'est-à-dire d'un sous-ensemble convexe de $\mathbb{R}^{2}$. On dit que $\subseteq$ est le support du polygone de Newton de $\mathcal{M}$. L'ensemble $\mathfrak{S}$ est la réunion des supports des cycles $\operatorname{Irr}\left(\widetilde{\Sigma}_{\Lambda}(r)(\mathcal{M})\right)$ et le polygone ne dépend que de ces cycles, on obtient donc immédiatement:

COROLLAIRE 2.2.10. Soit $\mathcal{M}$ un $\mathcal{D}_{X}$-module holonome tel que $Z$ est non $\{r\}$-microcaractéristique pour tout $r \geqslant 1$. Soit $\subseteq$ le support du polygone de Newton de $\mathcal{M}$.

Le support $\Im_{Z}$ du polygone de Newton de $\varphi^{*} \mathcal{M}$ est donné par: $\varsigma_{Z}=\varrho_{0} \varpi_{0}^{-1} \varsigma_{\text {et pour }}$ chaque composante $\mathfrak{S}_{Z}^{0}$ de $\mathfrak{\subseteq}_{Z}$ le polygone associé est la somme (au sens des sous-ensembles convexes de $\mathbb{R}^{2}$ ) des polygones associés à toutes les composantes $\varsigma^{0}$ de $\subseteq$ telles que $\Im_{Z}^{0}=\varrho_{0} \varpi_{0}^{-1} \varsigma^{0}$. 


\subsection{PENTES D'UN MODULE ET CROISSANCE DES SOLUTIONS}

L'égalité entre 'pentes algébriques' et 'pentes analytiques' d'un module holonome a été démontrée dans [9, théorème 2.4.3] par des méthodes géométriques. Montrons rapidement comment on peut retrouver ce théorème en utilisant les résultats précédents.

THÉORÈME 2.3.1. Soient $\mathcal{M}$ un module holonome, y un point de $Y$ et $r \in] 1,+\infty[$. Le nombre $r$ est une pente de $\mathcal{M}$ au point y si et seulement si:

$$
\mathbb{R} \mathcal{H} \operatorname{om}_{\mathcal{D}_{X}}\left(\mathcal{M}, \mathcal{B}_{Y \mid X}(r)\right) \neq \mathbb{R} \mathcal{H} \operatorname{om}_{\mathcal{D}_{X}}\left(\mathcal{M}, \mathcal{B}_{Y \mid X}\{r\}\right) \quad \text { au voisinage de } y
$$

Démonstration. Si $r$ n'est pas une pente, l'égalité entre les solutions a été montrée dans [7]. Ce que nous voulons montrer ici, c'est la réciproque. Nous utiliserons la proposition 2.2.6 plutôt que le théorème 2.2.8 sinon la démonstration ne serait pas indépendante de [9].

Montrons le résultat par récurrence sur la dimension de $X$, le cas de la dimension 1 étant bien connu depuis Ramis [11].

Soit $F$ le sous-ensemble discret de $Y$ de la remarque 2.2.5. Supposons tout d'abord que le point $y$ considéré n'appartient pas à $F$. Par définition de $F$, il existe une hypersurface $Z$ passant par $y$, transverse à $Y$, qui est non microcaractéristique pour tout $r$. Soit $f$ une équation de $Z$ (tout est local ici) et pour $\alpha \in \mathbb{C}$ soit $Z_{\alpha}=f^{-1}(\alpha)$. Si $\alpha$ est assez petit, la variété $Z_{\alpha}$ vérifie la même propriété que $Z$.

Si $r$ est une pente de $\mathcal{M}$, pour tout $\varepsilon>0$ il existe, d'après 2.2.6, $\alpha$ tel que $|\alpha|<\varepsilon$ et que $r$ soit une pente de $\mathcal{M}_{Z_{\alpha}}$. D'après l'hypothèse de récurrence, les complexes de solutions de $\mathcal{M}_{Z_{\alpha}}$ dans $\mathcal{B}_{Z_{\alpha} \cap Y \mid Z_{\alpha}}\{r\}$ et $\mathcal{B}_{Z_{\alpha} \cap Y \mid Z_{\alpha}}(r)$ sont différents et on conclut en appliquant le théorème de Cauchy-Kowalevska 2.2.4. que $\mathbb{R} \mathcal{H}$ om $_{\mathcal{D}_{X}}$ $\left(\mathcal{M}, \mathcal{B}_{Y \mid X}(r)\right) \neq \mathbb{R} \mathcal{H} \operatorname{om}_{\mathcal{D}_{X}}\left(\mathcal{M}, \mathcal{B}_{Y \mid X}\{r\}\right)$.

Supposons à présent que $y$ appartient à $F$ et que $r$ est une pente de $\mathcal{M}$. Notons $\Sigma_{0}$ la réunion des composantes irréductibles de $\Sigma_{\Lambda}(r)(\mathcal{M})$ non bihomogènes et dont l'adhérence de la projection sur $Y$ contient $y$. Par hypothèse $\Sigma_{0}$ n'est pas vide. Si la projection de $\Sigma_{0}$ sur $Y$ n'est pas réduite à $y$, dans tout voisinage de $y$ il $y$ a des points qui n'appartiennent pas à $F$ et on peut appliquer le résultat précédent.

On peut donc supposer qu'il existe un voisinage $V$ de $y$ tel que $\pi\left(\Sigma_{0}\right) \cap V=\{y\}$ avec $\pi$ projection canonique de $T^{*} \Lambda$ sur $Y$. En coordonnées, cela signifie que, sur $V, \Sigma_{0}$ est contenue dans $\left\{\left(x, \tau, x^{*}, \tau^{*}\right) \in T^{*} \Lambda \mid x=y\right\}$ et puisque elle est lagrangienne, elle est invariante en $x^{*}$ donc de la forme:

$$
\Sigma_{0}=\left\{\left(x, \tau, x^{*}, \tau^{*}\right) \in T^{*} \Lambda \mid x=y, \lambda\left(\tau, \tau^{*}\right)=0\right\}
$$

La variété $\Sigma_{0}$ est donc le produit du conormal à $y$ dans $Y$ par une surface $r$-homogène de $T^{*} \mathbb{C}^{2}$ d'équation $\lambda$. On vérifie facilement sur la définition que le cycle $\operatorname{Irr}\left(\Sigma_{\Lambda}(r)(\mathcal{M})\right)$ est nul si et seulement si la fonction $\lambda$ est bihomogène donc le cycle $\Sigma_{\Lambda}(r)(\mathcal{M})$ est bihomogène. Comme ce n'est pas le cas par hypothèse, le cycle 
$\operatorname{Irr}\left(\Sigma_{\Lambda}(r)(\mathcal{M})\right)$ n'est pas nul et d'après [7] cela implique que les solutions dans $\mathcal{B}_{Y \mid X}(r)$ et $\mathcal{B}_{Y \mid X}\{r\}$ sont différentes.

\section{Références}

1. Björk, J.-E: Rings of Differential Operators, North-Holland, Amsterdam, 1979.

2. Björk, J.-E.: Analytic D-Modules and Applications, Kluwer Acad. Publ., Dordrecht, 1993.

3. Kashiwara, M.: Systems of Microdifferential Equations, Progr. Math. 34, Birkhäuser, Basel, 1983.

4. Kashiwara, M.: Vanishing cycles and holonomic systems of differential equations, Lecture Notes in Math. 1016, Springer, New York, 1983, pp. 134-142.

5. Laurent, Y.: Théorie de la deuxième microlocalisation dans le domaine complex, Progr. Math. 53, Birkhäuser, Basel, 1985.

6. Laurent, Y.: Polygone de Newton et $b$-fonctions pour les modules microdifférentiels, Ann. Ecole Norm. Sup. (4) 20 (1987), 391-441.

7. Laurent, Y.: Vanishing cycles of irregular $\mathcal{D}$-modules, Compositio Math. 116 (1999), 241-310.

8. Laurent Y. and Malgrange, B.: Cycles proches, spécialisation et $\mathcal{D}$-modules, Ann. Inst. Fourier Grenoble 45 (1995).

9. Laurent, Y. and Mebkhout, Z.: Pentes algébriques et pentes analytiques d'un $\mathcal{D}$-module, Ann. Ecole Norm. Sup (4) 32 (1999), 39-69.

10. Mebkhout, Z.: Le théorème de positivité de l'irrégularité pour les $\mathcal{D}$-modules, In: The Grothendieck Festschrift III, Progr. Math. 88, Birkhäuser, Basel, 1990, pp. 83-132.

11. Ramis, J.-P: Théorèmes d'indices gevrey pour les équations différentielles ordinaires, Mem. Amer. Math. Soc. 48 (1984), No. 296.

12. Sato, M., Kawaï, T. and Kashiwara, M.: Hyperfunctions and pseudo-differential equations, In: Lecture Notes in Math. 287, Springer, New York, 1980, pp. 265-529.

13. Schapira, P.: Microdifferential Systems in the Complex Domain, Grundlehren Math. Wiss. 269, Springer, New York, 1985. 